Helsinki University of Technology Institute of Mathematics Research Reports

\title{
A POSTERIORI ERROR ANALYSIS FOR THE MORLEY PLATE ELEMENT WITH GENERAL BOUNDARY CONDITIONS
}

Lourenço Beirão da Veiga Jarkko Niiranen Rolf Stenberg 

Helsinki University of Technology Institute of Mathematics Research Reports

\section{A POSTERIORI ERROR ANALYSIS FOR THE MORLEY PLATE ELEMENT WITH GENERAL BOUNDARY CONDITIONS}

Lourenço Beirão da Veiga Jarkko Niiranen Rolf Stenberg 
Lourenço Beirão da Veiga, Jarkko Niiranen, Rolf Stenberg: A posteriori error analysis for the Morley plate element with general boundary conditions; Helsinki University of Technology Institute of Mathematics Research Reports A556 (2008).

\begin{abstract}
This paper introduces and analyses a local, residual based a posteriori error indicator for the Morley finite element method of the biharmonic Kirchhoff plate bending problem. In the theoretical part of the paper, a recent approach presented by the authors for clamped boundaries is extended to general boundary conditions. The error indicator is proven to be both reliable and efficient. The numerical part of the paper presents a set of results on various benchmark computations with different kinds of domains and boundary conditions. These tests verify the reliability and efficiency of the error estimator and illustrate the robustness of the method for adaptive mesh refinements.
\end{abstract}

AMS subject classifications: 65N30, 74S05, 74K20

Keywords: nonconforming finite elements, a posteriori error analysis, Morley plate element, Kirchhoff plate model, boundary conditions

\title{
Correspondence
}

beirao@mat.unimi.it, jarkko.niiranen@tkk.fi, rolf.stenberg@tkk.fi

ISBN 978-951-22-9602-6 (print)

ISBN 978-951-22-9603-3 (PDF)

ISSN 0784-3143 (print)

ISSN 1797-5867 (PDF)

Helsinki University of Technology

Faculty of Information and Natural Sciences

Department of Mathematics and Systems Analysis

P.O. Box 1100, FI-02015 TKK, Finland

email: math@tkk.fi http://math.tkk.fi/ 


\section{Introduction}

Plate structure models, together with other dimensionally reduced structure models as beams and shells, are the main building blocks in modern structural design. For thin plates, the most commonly used models in engineering applications are the Reissner-Mindlin and Kirchhoff-Love plates [13, 25, 16]. In modern engineering design, on the other hand, inreasing demands for accuracy and productivity have lead to exploitation of advanced computeraided design methodologies as adaptive finite element methods. In particular, adaptive methods provide an efficient and reliable way to decrease and control the discretization error of numerical approximations.

Regarding the finite element methods for the Kirchhoff-Love plate model, there exists various classical $[2,23,12]$ and more recent $[15,14,3,17,5,7]$ non-standard finite elements which avoid using high-order polynomial spaces of conforming, globally $C^{1}$-continuous elements $[12,10]$. The variety of a posteriori error analysis for Kirchhoff plate elements, instead, is still quite limited $[11,24,5,7,6]$. In addition, very often in rigorous finite element analysis for plates only clamped boundaries are considered. Some recent exceptions can be found in $[5,7,4,20]$.

In the present contribution, we complete our recent, theoretical a posteriori error analysis for the Morley plate element approximating the Kirchhoff plate problem with clamped boundaries [6]. We first extend our analysis to general boundary conditions, and then confirm the results by numerical benchmark computations. In particular, since a posteriori error analysis is intended to practical adaptive computations [19], it is natural to regard general boundary conditions and numerical results as a desirable target. The new features in the theoretical analysis of the present paper are not straightforward extensions of the results contained in [6]. Namely, for the reliability proof of the extended method, we need to introduce a new tensorial Helmholtz decomposition. Furthermore, a modification of a Clement type interpolation operator is needed for the reliability analysis as well.

The paper is organized as follows. In the next Section, we briefly recall the Kirchhoff plate bending problem and the Morley finite element formulation. In Section 3, we first introduce two interpolation operators and the Helmholtz decomposition, while in the following two subsections we prove, respectively, upper and lower bounds for our local error indicator. Finally, in Section 4, we present the numerical results.

In the Appendix, we recall a set of differential operators and formula for integration by parts, widely used throughout the text.

\section{Kirchhoff plate bending problem}

We consider the classical bending problem of an isotropic, linearly elastic plate. Let the undeformed midsurface of the plate be described by a polygonal domain $\Omega \subset \mathbb{R}^{2}$, with the boundary $\Gamma$. The plate is considered to be 
clamped on part $\Gamma_{C} \subset \Gamma$, simply supported on part $\Gamma_{S}$ and free on $\Gamma_{F}$ :

$$
\Gamma=\Gamma_{C} \cup \Gamma_{S} \cup \Gamma_{F} .
$$

We assume that $\Gamma_{C}, \Gamma_{S}, \Gamma_{F}$ are finite sums of connected components and that $\Gamma_{C}, \Gamma_{S}$ are given such that rigid body motions are avoided. Finally, a transverse load $F=G t^{3} f$ is applied, with $t$ denoting the thickness of the plate and $G$ denoting the shear modulus for the material.

\subsection{Continuous variational formulation}

Let $w$, the deflection of the plate, belong to the Sobolev space

$$
W=\left\{v \in H^{2}(\Omega) \mid v=0 \text { in } \Gamma_{C} \cup \Gamma_{S}, \nabla v \cdot \boldsymbol{n}=0 \text { in } \Gamma_{C}\right\},
$$

where we indicate with $\boldsymbol{n}$ the unit outward normal to the boundary $\Gamma$. Then, let the bilinear form for the problem be

$$
a(u, v)=(\boldsymbol{E} \varepsilon(\nabla u), \varepsilon(\nabla v))_{\Omega} \quad \forall u, v \in W,
$$

where the parentheses $(\cdot, \cdot)_{\Omega}$ above indicate the $L^{2}(\Omega)$ scalar product, and the fourth order positively definite elasticity tensor $\boldsymbol{E}$ is defined by the equation

$$
\boldsymbol{E} \boldsymbol{\sigma}=\frac{E}{12(1+\nu)}\left(\boldsymbol{\sigma}+\frac{\nu}{1-\nu} \operatorname{tr}(\boldsymbol{\sigma}) \boldsymbol{I}\right) \quad \forall \boldsymbol{\sigma} \in \mathbb{R}^{2 \times 2},
$$

with $E, \nu$ being the Young modulus and the Poisson ratio for the material, respectively.

Then, following the Kirchhoff plate bending model, the deflection $w$ of the plate can be found as the solution of the following variational problem: Find $w \in W$ such that

$$
a(w, v)=(f, v) \quad \forall v \in W .
$$

\subsection{Morley finite element formulation}

First, let a regular family of triangular meshes $\left\{\mathcal{C}_{h}\right\}_{h}$ be given on the domain $\Omega$. We assume that the mesh family is consistent with the boundary conditions, in the sense that all boundary edges of the triangulation fall entirely either in $\Gamma_{C}, \Gamma_{S}$ or $\Gamma_{F}$.

In what follows, we will indicate by $h_{K}$ the diameter of each element $K$, while $h$ will indicate the maximum size of all the elements in the mesh. With $\mathcal{E}_{h}$, we will indicate the set of all the edges, with $\mathcal{E}_{h}^{i}$ a subset comprising only the internal edges, while $\mathcal{E}_{h}^{c}, \mathcal{E}_{h}^{s}, \mathcal{E}_{h}^{f}$ indicate the set of edges on $\Gamma_{C}, \Gamma_{S}, \Gamma_{F}$, respectively. Given any $e \in \mathcal{E}_{h}$, the scalar $h_{e}$ will represent its length. Finally, to each edge $e \in \mathcal{E}_{h}$ we associate a normal unit vector $\boldsymbol{n}_{e}$ and a tangent unit vector $\boldsymbol{s}_{e}$, the latter given by a counter clockwise $90^{0}$ rotation of the former. The choice of the particular normal is arbitrary, although fixed once and for all, for internal edges, while we assume that $\boldsymbol{n}_{e}=\boldsymbol{n}$ for boundary edges. 
Furthermore, we will also need the definition of jumps: Let $K_{+}$and $K_{-}$ be any two triangles with an edge $e$ in common, such that the unit outward normal to $K_{-}$at $e$ corresponds to $\boldsymbol{n}_{e}$. Furthermore, given a piecewise continuous scalar function $v$ on $\Omega$, call $v^{+}$(respectively $v^{-}$) the trace $\left.v\right|_{K_{+}}$ (respectively $\left.v\right|_{K_{-}}$) on $e$. Then, the jump of $v$ across the edge $e$ is a scalar function living on $e$, given by

$$
\llbracket v \rrbracket=v^{+}-v^{-} .
$$

For a vector-valued function, also the jump is vector-valued, defined as above component by component. Finally, the jump on boundary edges is simply given by the trace of the function on each edge.

Next, the discrete Morley space [23] is introduced as

$$
W_{h}=\left\{v \in M_{2, h} \mid \int_{e} \llbracket \nabla v \cdot \boldsymbol{n}_{e} \rrbracket=0 \quad \forall e \in \mathcal{E}_{h}^{i} \cup \mathcal{E}_{h}^{c}\right\},
$$

where $M_{2, h}$ denotes the space of second order piecewise polynomial functions on $\mathcal{C}_{h}$ which are continuous at the vertices of all the internal triangles and zero at all the vertices of $\Gamma_{C} \cup \Gamma_{S}$.

Finally, the Morley finite element approximation of the variational problem (2.5) reads as follows:

Method 1. Find $w_{h} \in W_{h}$ such that

$$
a_{h}\left(w_{h}, v_{h}\right)=\left(f, v_{h}\right) \quad \forall v_{h} \in W_{h},
$$

where

$$
a_{h}\left(u_{h}, v_{h}\right)=\sum_{K \in \mathcal{C}_{h}}\left(\boldsymbol{E} \varepsilon\left(\nabla u_{h}\right), \varepsilon\left(\nabla v_{h}\right)\right)_{K} \quad \forall u_{h}, v_{h} \in W_{h} .
$$

We note that the bilinear form $a_{h}$ is positive definite on the space $W_{h}$. Therefore, there exists a unique solution to the problem (2.8).

We further introduce the discrete norm

$$
\begin{aligned}
\|\| v\|\|_{h}^{2}= & \sum_{K \in \mathcal{C}_{h}}|v|_{H^{2}(K)}^{2}+\sum_{e \in \mathcal{E}_{h}^{i} \cup \mathcal{E}_{h}^{c} \cup \mathcal{E}_{h}^{s}} h_{e}^{-3}\|\llbracket v \rrbracket\|_{L^{2}(e)}^{2} \\
& +\sum_{e \in \mathcal{E}_{h}^{i} \cup \mathcal{E}_{h}^{c}} h_{e}^{-1}\left\|\llbracket \nabla v \cdot \boldsymbol{n}_{e} \rrbracket\right\|_{L^{2}(e)}^{2}
\end{aligned}
$$

on the space $W_{h}+H^{2}$. Then the stability and convergence of Method 1 in the norm (2.10) is well established, as proved for the clamped case in [27, 22]:

Proposition 1. Assuming that $\Gamma=\Gamma_{C}$ let $w$ be the solution of the problem (2.5) and $w_{h}$ the solution of the problem (2.8). Then there exists a positive constant $C$, independent of $h$, such that

$$
\left\|\left|w-w_{h}\right|\right\|_{h} \leq C h\left(|w|_{H^{3}(\Omega)}+h\|f\|_{L^{2}(\Omega)}\right) .
$$

The numerical results in Section 4 indicate the same convergence rate for general boundary conditions as well. Hence, the corresponding a priori analysis is omitted here. 


\section{A posteriori error estimates}

In this section, we derive lower and upper bounds for our a posteriori error indicator of the Morley element, extending the results of [6] to the case of general boundary conditions. After some preliminaries, we prove the reliability and efficiency results of the error estimator

$$
\eta=\left(\sum_{K \in \mathcal{C}_{h}} \eta_{K}^{2}\right)^{1 / 2}
$$

where

$$
\begin{aligned}
\eta_{K}^{2}= & h_{K}^{4}\left\|f_{h}\right\|_{L^{2}(K)}^{2}+\sum_{e \in \partial K^{\prime}} c_{e} h_{e}^{-3}\left\|\llbracket w_{h} \rrbracket\right\|_{L^{2}(e)}^{2} \\
& +\sum_{e \in \partial K^{\prime \prime}} c_{e} h_{e}^{-1}\left\|\llbracket \nabla w_{h} \cdot \boldsymbol{n}_{e} \rrbracket\right\|_{L^{2}(e)}^{2}
\end{aligned}
$$

with the compact notation

$$
\begin{aligned}
& \partial K^{\prime}=\partial K \cap\left\{\mathcal{E}_{h}^{i} \cup \mathcal{E}_{h}^{c} \cup \mathcal{E}_{h}^{s}\right\}, \\
& \partial K^{\prime \prime}=\partial K \cap\left\{\mathcal{E}_{h}^{i} \cup \mathcal{E}_{h}^{c}\right\} .
\end{aligned}
$$

Moreover, $f_{h}$ denotes, as usual, some approximation of $f$, while $c_{e}=1 / 2$ if $e \in \mathcal{E}_{h}^{i}$ and $c_{e}=1$ otherwise.

\subsection{A pair of interpolation operators}

In this subsection, we introduce two interpolation operators which will be needed in prooving the reliability of our estimator. We start by introducing the first one:

Definition 1. Given any $v \in W$, we indicate with $v_{I}$ the only function in $W_{h}$ such that

$$
\begin{aligned}
& v_{I}(p)=v(p) \quad \text { for every vertex } p \text { of the mesh } \mathcal{C}_{h}, \\
& \int_{e}\left(\nabla v-\nabla v_{I}\right) \cdot \boldsymbol{n}_{e}=0 \quad \forall e \in \mathcal{E}_{h} .
\end{aligned}
$$

This interpolant has the following approximation property:

$$
\left\|v-v_{I}\right\|_{L^{2}(K)} \leq C h_{K}^{2}|v|_{H^{2}(K)} \quad \forall K \in \mathcal{C}_{h}, v \in W .
$$

Moreover, integration by parts gives

$$
\int_{e}\left(\nabla v-\nabla v_{I}\right) \cdot \boldsymbol{s}_{e}=0 \quad \forall e \in \mathcal{E}_{h} .
$$

In order to present the second interpolant, we next introduce two auxiliary interpolation operators $\Pi_{B}, \Pi_{C}$, the building blocks of our second interpolant. 
Auxiliary operator $\Pi_{C}$. We start briefly reviewing the Clement type interpolant of Scott and Zhang [26]. This interpolant we shall modify below in order to construct a similar interpolant with some additional features needed for our purposes.

To each node $\nu$ of the mesh $\mathcal{C}_{h}$, we associate an edge $e(\nu)$ such that $\nu \in e$. Then, for each node $\nu$ we introduce a linear scalar function $f_{\nu}: e(\nu) \rightarrow \mathbb{R}$ such that

$$
\int_{e(\nu)} f_{\nu}(s) p(s) \mathrm{d} s=p(\nu) \quad \forall p \in P_{1}(e)
$$

where $s$ stands for the standard coordinate along the edge $e$ and $P_{1}(e)$ represents the space of first order polynomials on the edge. It is easy to check that such scalar functions exist.

Let now $M_{1}^{0}$ indicate the space of piecewise linear continuous functions on $\mathcal{C}_{h}$. Then, given any $v \in H^{1}(\Omega)$, we define its Scott-Zhang interpolant as

$$
\Pi(v)=\sum_{\nu \in \mathcal{V}_{h}} \varphi_{\nu} \int_{e(\nu)} f_{\nu}(s) v(s) \mathrm{d} s,
$$

where $\mathcal{V}_{h}$ represents the set of mesh nodes and $\varphi_{\nu} \in M_{1}^{0}$ is the standard nodal basis function for the general node $\nu$. Note that it is equivalent to define $\Pi(v)$ as the only function in $M_{1}^{0}$ such that

$$
(\Pi(v))(\nu)=\int_{e(\nu)} f_{\nu}(s) v(s) \mathrm{d} s \quad \forall \nu \in \mathcal{V}_{h}
$$

This interpolant satisfies the classical approximation properties of the Clement type, see [26], i.e.,

$$
\begin{aligned}
& \|v-\Pi(v)\|_{H^{m}(K)} \leq C h_{K}^{1-m}\|v\|_{H^{1}(\tilde{K})} \quad \forall K \in \mathcal{C}_{h}, m=0,1, \\
& \|v-\Pi(v)\|_{L^{2}(e)} \leq C h_{K}^{1 / 2}\|v\|_{H^{1}(\tilde{K})} \quad \forall e \in \partial K, K \in \mathcal{C}_{h},
\end{aligned}
$$

where $\tilde{K}$ indicates the set of all the triangles of $\mathcal{C}_{h}$ with a nonempty intersection with $K \in \mathcal{C}_{h}$.

Moreover, the operator $\Pi$ has the following fundamental property: for all $\nu \in \mathcal{V}_{h}$ and $v \in H^{1}(\Omega)$

$$
\left.v\right|_{e(\nu)} \in P_{1}(e(\nu)) \Longrightarrow(\Pi(v))(\nu)=v(\nu) .
$$

We will now introduce the vector interpolant $\Pi_{C}$ similar to the operator $\Pi$ above, with a particular property which will be useful below. We start by giving the rule by which edges are associated to nodes.

Assignment rule for $\Pi_{C}$. If a node $\nu \in e^{\prime}$ for some $e^{\prime} \in \mathcal{E}_{h}^{f}$, then $e(\nu)=e^{\prime}$, and an arbitrary edge can be chosen if more than one fulfill this property. Otherwise, if $\nu \in e^{\prime}$ for some $e^{\prime} \in \mathcal{E}_{h}^{s}$, then $e(\nu)=e^{\prime}$, where again any edge can be chosen among those that fulfill this property. Regarding the rest of the nodes, the only restriction is that boundary nodes must be associated to boundary edges and, obviously, $\nu \in e(\nu)$. 
For the sake of exposition, we assume at first that all connected components of $\Gamma_{S}$ are straight, i.e. without angles. Then, for each function $\boldsymbol{v} \in\left[H^{1}(\Omega)\right]^{2}$, we simply set $\Pi_{C}(\boldsymbol{v})$ as the only piecewise linear continuous vector field that satisfies

$$
\left(\Pi_{C}(\boldsymbol{v})\right)_{j}(\nu)=\int_{e(\nu)} f_{\nu}(s) v_{j}(s) \mathrm{d} s \quad \forall \nu \in \mathcal{V}_{h}, j=1,2,
$$

where a subindex $j$ represents the respective vector component. Note that $\Pi_{C}$ is nothing but a componentwise combination of $\Pi$.

In the presence of angles inside connected components of $\Gamma_{S}$, the value of $\Pi_{C}(\boldsymbol{v})$ at those angle nodes must be changed. Given $\nu^{\prime}$ any such angle node, let $e_{1}$ with normal $\boldsymbol{n}_{1}$ and $e_{2}$ with normal $\boldsymbol{n}_{2}$ be the two edges in $\Gamma_{S}$ connecting in $\nu^{\prime}$. We then modify the value of $\Pi_{C}(\boldsymbol{v})$ in $\nu^{\prime}$, by setting

$$
\Pi_{C}(\boldsymbol{v}) \cdot \boldsymbol{n}_{i}\left(\nu^{\prime}\right)=\int_{e_{i}} f_{\nu^{\prime}}(s) \boldsymbol{v}(s) \cdot \boldsymbol{n}_{i} \mathrm{~d} s \quad i=1,2 .
$$

Since $\boldsymbol{n}_{1} \neq \boldsymbol{n}_{2}, \Pi_{C}(\boldsymbol{v})\left(\nu^{\prime}\right)$ is well defined. It is simple to check that $\Pi_{C}$ inherits from $\Pi$ the Clement approximation properties

$$
\begin{aligned}
& \left\|\boldsymbol{v}-\Pi_{C}(\boldsymbol{v})\right\|_{H^{m}(K)} \leq C h_{K}^{1-m}\|\boldsymbol{v}\|_{H^{1}(\tilde{K})} \quad \forall K \in \mathcal{C}_{h}, m=0,1, \\
& \left\|\boldsymbol{v}-\Pi_{C}(\boldsymbol{v})\right\|_{L^{2}(e)} \leq C h_{K}^{1 / 2}\|\boldsymbol{v}\|_{H^{1}(\tilde{K})} \quad \forall e \in \partial K, K \in \mathcal{C}_{h} .
\end{aligned}
$$

Moreover, essentially due to the linearity of $\Pi_{C}$ and (3.14), it can be showed that the following property holds:

$$
\begin{aligned}
& \boldsymbol{v} \in\left[P_{1}(e)\right]^{2} \quad \forall e \in \Gamma_{F}, \boldsymbol{v} \cdot \boldsymbol{n} \in P_{1}(e) \quad \forall e \in \Gamma_{S} \\
& \Longrightarrow \Pi_{C} \boldsymbol{v}=\boldsymbol{v} \text { on } \Gamma_{F}, \Pi_{C} \boldsymbol{v} \cdot \boldsymbol{n}=\boldsymbol{v} \cdot \boldsymbol{n} \text { on } \Gamma_{S} .
\end{aligned}
$$

Auxiliary operator $\Pi_{B}$. Given any edge $e \in \mathcal{E}_{h}$, let $B_{e}$ indicate the globally continuous, piecewise second order polynomial function which is equal to one at the midpoint of $e$ and zero at all the other vertices and other edge midpoints of the mesh. Let $V_{B}^{\prime}$ indicate the discrete space given by the span of all $B_{e}, e \in \mathcal{E}_{h}$. Furthermore, call $V_{B}$ the discrete vector field given by two copies of the space $V_{B}^{\prime}$, one for each component. We then introduce the operator $\Pi_{B}$ defined by

$$
\Pi_{B}:\left[H^{1}(\Omega)\right]^{2} \rightarrow V_{B}, \int_{e}\left(\boldsymbol{v}-\Pi_{B}(\boldsymbol{v})\right)=\mathbf{0} \quad \forall e \in \mathcal{E}_{h} .
$$

Using the definition (3.20), inverse inequalities and the Agmon inequality [1], it is easy to check that $\Pi_{B}$ satisfies the following property for all $v \in$ $H^{1}(\Omega)$ :

$$
\left\|\Pi_{B}(\boldsymbol{v})\right\|_{H^{m}(K)} \leq C h_{K}^{1-m}\left(h_{K}^{-1}\|\boldsymbol{v}\|_{L^{2}(K)}+|\boldsymbol{v}|_{H^{1}(K)}\right) \quad \forall K \in \mathcal{C}_{h} .
$$

Then, we are able to introduce our second interpolant: 
Definition 2. Given any $\boldsymbol{v} \in\left[H^{1}(\Omega)\right]^{2}$, we indicate with $\boldsymbol{v}_{I I}$ the continuous piecewise polynomial function of second order given by

$$
\boldsymbol{v}_{I I}=\Pi_{C}(\boldsymbol{v})+\Pi_{B}\left(\boldsymbol{v}-\Pi_{C}(\boldsymbol{v})\right) .
$$

Using the properties (3.17), (3.18) and (3.21) we easily get

$$
\left\|\boldsymbol{v}-\boldsymbol{v}_{I I}\right\|_{H^{m}(K)} \leq C h_{K}^{1-m}\|\boldsymbol{v}\|_{H^{1}(\tilde{K})} \quad \forall K \in \mathcal{C}_{h}, m=0,1
$$

for all $\boldsymbol{v} \in\left[H^{1}(\Omega)\right]^{2}$. Moreover, directly from (3.20) and Definition 2, it follows

$$
\int_{e}\left(\boldsymbol{v}-\boldsymbol{v}_{I I}\right)=\mathbf{0} \quad \forall e \in \mathcal{E}_{h}, \boldsymbol{v} \in\left[H^{1}(\Omega)\right]^{2} .
$$

Finally, we note that property (3.19) holds identically also for the new interpolant of Definition 2, since along the boundary edges of $\Gamma_{F}$ (respectively $\left.\Gamma_{S}\right)$ the bubble part (respectively the bubble part in the normal direction) vanishes due to (3.22), (3.19) and the definition of the operator $\Pi_{B}$. We therefore have

$$
\begin{aligned}
& \boldsymbol{v} \in\left[P_{1}(e)\right]^{2} \quad \forall e \in \Gamma_{F}, \boldsymbol{v} \cdot \boldsymbol{n} \in P_{1}(e) \quad \forall e \in \Gamma_{S} \\
& \Longrightarrow \boldsymbol{v}_{I I}=\boldsymbol{v} \text { on } \Gamma_{F}, \boldsymbol{v}_{I I} \cdot \boldsymbol{n}=\boldsymbol{v} \cdot \boldsymbol{n} \text { on } \Gamma_{S} .
\end{aligned}
$$

Remark 1. We note that the constants $C$ appearing in (3.17) and (3.18) are not uniform in the angular width of a simply supported angle. Namely, when such an angle tends towards the straight angle, i.e., the angle width $\pi$, the constants $C$ may explode to infinity. On one hand, for any given polygonal domain, all angles are fixed and therefore the constants are bounded independently of the mesh size. On the other hand, the latter observation is not true if a simply supported domain with a smooth boundary is approximated by a series of adaptively refined polygonal domains. Nevertheless, this case would also need an estimator for the error of the geometry approximation and it is therefore beyond the scope of the present paper.

\subsection{A Helmholtz decomposition lemma}

In this section, we extend the tensorial Helmholtz decomposition lemma of [6] to general boundary conditions. The differential operators used below are defined in the Appendix. Note that the proof of Lemma 1 is almost identical to that of [6], while the differences with respect to the clamped case are shown in Corollary 1.

Let the space $\tilde{H}^{m}(\Omega), m \in \mathbb{N}$, indicate the quotient space of $H^{m}(\Omega)$ where the seminorm $|\cdot|_{H^{m}(\Omega)}$ is null. Moreover, let $L_{0}^{2}(\Omega)$ indicate, as usual, the space of functions in $L^{2}(\Omega)$ with zero average over $\Omega$.

Lemma 1. Let $\boldsymbol{\sigma}$ be a second order tensor field in $L^{2}\left(\Omega ; \mathbb{R}^{2 \times 2}\right)$. Then, there exist $\psi \in W, \rho \in L_{0}^{2}(\Omega)$ and $\phi \in\left[\tilde{H}^{1}(\Omega)\right]^{2}$ such that

$$
\boldsymbol{\sigma}=\boldsymbol{E} \varepsilon(\nabla \psi)+\boldsymbol{\rho}+\operatorname{Curl} \boldsymbol{\phi},
$$


where the second order tensor

$$
\boldsymbol{\rho}=\left(\begin{array}{cc}
0 & -\rho \\
\rho & 0
\end{array}\right)
$$

Moreover,

$$
\|\psi\|_{H^{2}(\Omega)}+\|\rho\|_{L^{2}(\Omega)}+\|\phi\|_{H^{1}(\Omega)} \leq C\|\boldsymbol{\sigma}\|_{L^{2}(\Omega)} .
$$

Proof. Let $\psi$ be the solution of the following problem:

Find $\psi \in W$ such that

$$
(\boldsymbol{E} \boldsymbol{\varepsilon}(\nabla \psi), \boldsymbol{\varepsilon}(\nabla v))=(\boldsymbol{\sigma}, \boldsymbol{\varepsilon}(\nabla v)) \quad \forall v \in W .
$$

The problem above has a unique solution due to the coercivity of the considered bilinear forms on the respective spaces. The rest of the proof is identical to the proof of Lemma 1 in [6].

We have the following corollary concerning the general boundary conditions:

Corollary 1. Let $\phi$ be the function introduced in Lemma 1. Then it holds

$$
\begin{array}{ll}
{[\operatorname{Curl} \phi]_{n \boldsymbol{n}}:=[(\operatorname{Curl} \boldsymbol{\phi}) \boldsymbol{n}] \cdot \boldsymbol{n}=0} & \text { on } \Gamma_{S} \cup \Gamma_{F} \\
{[\operatorname{Curl} \boldsymbol{\phi}]_{\boldsymbol{n} \boldsymbol{s}}:=[(\operatorname{Curl} \boldsymbol{\phi}) \boldsymbol{n}] \cdot \boldsymbol{s}=c_{i}} & \text { on } \Gamma_{F}^{i}, i=1,2, \ldots, m,
\end{array}
$$

where $c_{i} \in \mathbb{R}$ and $\Gamma_{F}^{i}, i=1,2, \ldots, m$, represent the $m$ connected components of $\Gamma_{F}$.

Proof. A straightforward calculation, using $\boldsymbol{s} \cdot \boldsymbol{s}=1$ and $\boldsymbol{s} \cdot \boldsymbol{n}=0$, gives on the boundary $\Gamma$

$$
[\boldsymbol{\rho}]_{n \boldsymbol{n}}=0, \quad[\boldsymbol{\rho}]_{\boldsymbol{n} \boldsymbol{s}}=\rho,
$$

to be interpreted in the sense of traces.

Standard arguments in variational analysis and integration by parts in (3.29) easily give

$$
\begin{aligned}
& \langle(\boldsymbol{E} \boldsymbol{\varepsilon}(\nabla \psi)-\boldsymbol{\sigma}) \cdot \boldsymbol{n}, \nabla v\rangle_{\Gamma} \\
& -\langle\operatorname{div}(\boldsymbol{E} \boldsymbol{\varepsilon}(\nabla \psi)-\boldsymbol{\sigma}) \cdot \boldsymbol{n}, v\rangle_{\Gamma}=0 \quad \forall v \in W,
\end{aligned}
$$

where here and in the sequel $\langle,\rangle_{\Gamma}$ is to be intended in the sense of traces and dualities (see the Appendix). Note that the identity (3.33) can be rewritten, through a normal and tangent component splitting, as

$$
\begin{aligned}
& \left\langle[\boldsymbol{E} \boldsymbol{\varepsilon}(\nabla \psi)-\boldsymbol{\sigma}]_{\boldsymbol{n} \boldsymbol{n}}, \nabla v \cdot \boldsymbol{n}\right\rangle_{\Gamma} \\
& +\left\langle[\boldsymbol{E} \boldsymbol{\varepsilon}(\nabla \psi)-\boldsymbol{\sigma}]_{\boldsymbol{n} \boldsymbol{s}}, \nabla v \cdot \boldsymbol{s}\right\rangle_{\Gamma} \\
& -\langle\operatorname{div}(\boldsymbol{E} \varepsilon(\nabla \psi)-\boldsymbol{\sigma}) \cdot \boldsymbol{n}, v\rangle_{\Gamma}=0 \quad \forall v \in W .
\end{aligned}
$$


Testing with $v \in W$ being zero on $\Gamma$ one obtains from (3.34)

$$
[\boldsymbol{E} \boldsymbol{\varepsilon}(\nabla \psi)-\boldsymbol{\sigma}]_{\boldsymbol{n} \boldsymbol{n}}=0 \quad \text { on } \Gamma_{S} \cup \Gamma_{F},
$$

which together with the first identity in (3.32) and recalling (3.26) gives (3.30).

For (3.31), we now observe that, by first applying the operator div to (3.26) and then recalling the definition (3.27), it follows

$$
\begin{aligned}
& \operatorname{div}(\boldsymbol{E} \boldsymbol{\varepsilon}(\nabla \psi)-\boldsymbol{\sigma}) \cdot \boldsymbol{n}=-(\operatorname{div} \boldsymbol{\rho}) \cdot \boldsymbol{n}=-(\operatorname{curl} \rho) \cdot \boldsymbol{n} \\
& =\nabla \rho \cdot \boldsymbol{s}=\frac{\partial \rho}{\partial s}
\end{aligned}
$$

where here and in the sequel $s$ indicates a coordinate along the boundary. Due to (3.35), the first term in (3.34) vanish. For the sake of exposition, we at first neglect the presence of angles in $\Gamma_{F}^{i}$ by assuming that each $\Gamma_{F}^{i}$ is straight. In such case, by recalling (3.36) and integrating by parts separately along each $\Gamma_{F}^{i}$ in (3.34), one obtains

$$
\left\langle\frac{\partial}{\partial s}[\boldsymbol{E} \boldsymbol{\varepsilon}(\nabla \psi)-\boldsymbol{\sigma}]_{n \boldsymbol{s}}+\frac{\partial \rho}{\partial s}, v\right\rangle_{\Gamma}=0 \quad \forall v \in W .
$$

Due to the second identity in (3.32) and the definition (3.26), we get from (3.37)

$$
\left\langle\frac{\partial}{\partial s}[\operatorname{Curl} \phi]_{n \boldsymbol{s}}, v\right\rangle_{\Gamma}=0 \quad \forall v \in W
$$

which clearly implies (3.31).

Whenever an angle inside $\Gamma_{F}^{i}$ exists for some $i$, we follow the same identical argument as above separately on each straight component of $\Gamma_{F}^{i}$. Let $\Gamma_{F}^{i, j}$, $j=1,2, \ldots, m_{i}$ represent the straight components such that

$$
\Gamma_{F}^{i}=\cup_{j=1}^{m_{i}} \Gamma_{F}^{i, j} .
$$

Testing with functions $v$ being zero at the endpoints of each $\Gamma_{F}^{i, j}$ we then get

$$
[\operatorname{Curl} \phi]_{n \boldsymbol{s}}=c_{i, j} \quad \text { on } \Gamma_{F}^{i, j}, j=1,2, \ldots, m_{i},
$$

with $c_{i, j} \in \mathbb{R}, j=1,2, \ldots, m_{i}$. We finally get that all $c_{i, j}$ are equal, i.e.

$$
c_{i, j}=c_{i} \quad \forall j=1,2, \ldots, m_{i},
$$

simply by testing with functions $v$ which are non-zero at the angles.

\subsection{Reliability}

We have the following lower bound for the error estimator:

Theorem 1. Let $w$ be the solution of the problem (2.5) and $w_{h}$ the solution of the problem (2.8). Then it holds

$$
\left\|\mid w-w_{h}\right\| \|_{h} \leq C\left(\sum_{K \in \mathcal{C}_{h}} \eta_{K}^{2}+\sum_{K \in \mathcal{C}_{h}} h_{K}^{4}\left\|f-f_{h}\right\|_{L^{2}(K)}^{2}\right)^{1 / 2} .
$$


Proof. Recalling that $w \in W$, it immediately follows

$$
\begin{aligned}
\left\|\left|w-w_{h} \|\right|_{h}^{2}=\right. & \sum_{K \in \mathcal{C}_{h}}\left|w-w_{h}\right|_{H^{2}(K)}^{2}+\sum_{e \in \mathcal{E}_{h}^{i} \cup \mathcal{E}_{h}^{c} \cup \mathcal{E}_{h}^{s}} h_{e}^{-3}\left\|\llbracket w_{h} \rrbracket\right\|_{L^{2}(e)}^{2} \\
& +\sum_{e \in \mathcal{E}_{h}^{i} \cup \mathcal{E}_{h}^{c}} h_{e}^{-1}\left\|\llbracket \nabla w_{h} \cdot \boldsymbol{n}_{e} \rrbracket\right\|_{L^{2}(e)}^{2} .
\end{aligned}
$$

Therefore, due to the definition of $\eta_{K}$ in (3.2) and the norm (2.10), what needs to be proved is

$$
\sum_{K \in \mathcal{C}_{h}}\left|w-w_{h}\right|_{H^{2}(K)}^{2} \leq C\left(\sum_{K \in \mathcal{C}_{h}} \eta_{K}^{2}+\sum_{K \in \mathcal{C}_{h}} h_{K}^{4}\left\|f-f_{h}\right\|_{L^{2}(K)}^{2}\right) .
$$

For convenience, we divide the proof of (3.44) into five steps.

Step 1. Let in the sequel $e_{h}$ represent the error $w-w_{h}$. First due to the positive definiteness and symmetry of the fourth order tensor $\boldsymbol{E}$, then applying Lemma 1 to the tensor field $\boldsymbol{E} \boldsymbol{\varepsilon}\left(\nabla e_{h}\right)$, we have

$$
\begin{aligned}
\sum_{K \in \mathcal{C}_{h}}\left|e_{h}\right|_{H^{2}(K)}^{2} & \leq C a_{h}\left(e_{h}, e_{h}\right) \\
& =\sum_{K \in \mathcal{C}_{h}}\left(\varepsilon\left(\nabla e_{h}\right), \boldsymbol{E} \boldsymbol{\varepsilon}\left(\nabla e_{h}\right)\right)_{K}=T_{1}+T_{2}+T_{3},
\end{aligned}
$$

where

$$
\begin{aligned}
& T_{1}=\sum_{K \in \mathcal{C}_{h}}\left(\varepsilon\left(\nabla e_{h}\right), \boldsymbol{E} \varepsilon(\nabla \psi)\right)_{K}, \\
& T_{2}=\sum_{K \in \mathcal{C}_{h}}\left(\varepsilon\left(\nabla e_{h}\right), \boldsymbol{\rho}\right)_{K}, \\
& T_{3}=\sum_{K \in \mathcal{C}_{h}}\left(\varepsilon\left(\nabla e_{h}\right), \operatorname{Curl} \phi\right)_{K} .
\end{aligned}
$$

We note that, by recalling (3.28), it holds

$$
\|\psi\|_{H^{2}(\Omega)}^{2}+\|\boldsymbol{\phi}\|_{H^{1}(\Omega)}^{2} \leq C \sum_{K \in \mathcal{C}_{h}}\left|e_{h}\right|_{H^{2}(K)}^{2} .
$$

Step 2. We now bound the three terms $T_{1}, T_{2}, T_{3}$ above. Due to the symmetry of $\boldsymbol{E}$, from (2.5) we get

$$
T_{1}=(f, \psi)_{\Omega}-\sum_{K \in \mathcal{C}_{h}}\left(\boldsymbol{E} \varepsilon\left(\nabla w_{h}\right), \varepsilon(\nabla \psi)\right)_{K} .
$$

Let now $\psi_{I} \in W_{h}$ be the approximation of $\psi$ defined in Definition 1. Recalling (2.8) and integrating by parts on each triangle, from (3.50) it follows

$$
\begin{aligned}
T_{1} & =\left(f, \psi-\psi_{I}\right)_{\Omega}-\sum_{K \in \mathcal{C}_{h}}\left(\boldsymbol{E} \boldsymbol{\varepsilon}\left(\nabla w_{h}\right), \boldsymbol{\varepsilon}\left(\nabla\left(\psi-\psi_{I}\right)\right)\right)_{K} \\
& =\left(f, \psi-\psi_{I}\right)_{\Omega}-\sum_{K \in \mathcal{C}_{h}} \sum_{e \in \partial K}\left\langle\boldsymbol{E} \boldsymbol{\varepsilon}\left(\nabla w_{h}\right) \boldsymbol{n}_{K}, \nabla\left(\psi-\psi_{I}\right)\right\rangle_{e},
\end{aligned}
$$


where, here and in the sequel, $\boldsymbol{n}_{K}$ indicates the outward unit normal to each edge of $K \in \mathcal{C}_{h}$.

Observing that $\boldsymbol{E} \boldsymbol{\varepsilon}\left(\nabla w_{h}\right) \boldsymbol{n}_{K}$ is constant on each edge, then the properties (3.6) and (3.8) applied to (3.51) imply

$$
T_{1}=\left(f, \psi-\psi_{I}\right)_{\Omega}=\left(f-f_{h}, \psi-\psi_{I}\right)_{\Omega}+\left(f_{h}, \psi-\psi_{I}\right)_{\Omega} .
$$

Two Hölder inequalities and the interpolation property (3.7) therefore give

$$
T_{1} \leq C\left(\sum_{K \in \mathcal{C}_{h}} h_{K}^{4}\left\|f-f_{h}\right\|_{L^{2}(K)}^{2}+\sum_{K \in \mathcal{C}_{h}} h_{K}^{4}\left\|f_{h}\right\|_{L^{2}(K)}^{2}\right)^{1 / 2}\|\psi\|_{H^{2}(\Omega)} .
$$

Regarding the term $T_{2}$, it is sufficient to observe that, due to the symmetry of $\boldsymbol{\varepsilon}\left(\nabla e_{h}\right)$ and the definition of $\boldsymbol{\rho}$ in (3.27), it immediately follows

$$
T_{2}=\sum_{K \in \mathcal{C}_{h}}\left(\varepsilon\left(\nabla e_{h}\right), \boldsymbol{\rho}\right)_{K}=0 .
$$

Step 3. We now bound the term $T_{3}$ in (3.48). Recalling that $w \in W$ and the fact that $\operatorname{div} \operatorname{Curl} \phi=\mathbf{0}$, integration by parts (see the Appendix) for the $w$ part in $T_{3}$ gives

$$
\begin{aligned}
& (\boldsymbol{\varepsilon}(\nabla w), \operatorname{Curl} \phi)_{\Omega}=\langle\nabla w, \operatorname{Curl} \boldsymbol{\phi} \boldsymbol{n}\rangle_{\Gamma} \\
& =\left\langle\nabla w \cdot \boldsymbol{n},[\operatorname{Curl} \phi]_{\boldsymbol{n} \boldsymbol{n}}\right\rangle_{\Gamma}+\left\langle\nabla w \cdot \boldsymbol{s},[\operatorname{Curl} \boldsymbol{\phi}]_{\boldsymbol{n} \boldsymbol{s}}\right\rangle_{\Gamma}= \\
& =\left\langle\nabla w \cdot \boldsymbol{n},[\operatorname{Curl} \phi]_{\boldsymbol{n} \boldsymbol{n}}\right\rangle_{\Gamma_{S} \cup \Gamma_{F}}+\left\langle\nabla w \cdot \boldsymbol{s},[\operatorname{Curl} \boldsymbol{\phi}]_{\boldsymbol{n} \boldsymbol{s}}\right\rangle_{\Gamma_{F}} .
\end{aligned}
$$

From identity (3.55), using Corollary 1, we get

$$
(\varepsilon(\nabla w), \operatorname{Curl} \phi)_{\Omega}=\sum_{i=1}^{m}\left\langle\nabla w \cdot s, c_{i}\right\rangle_{\Gamma_{F}^{i}} .
$$

Integration by parts edge by edge in (3.56) now gives

$$
\begin{aligned}
& (\varepsilon(\nabla w), \operatorname{Curl} \phi)_{\Omega} \\
& =\sum_{i=1}^{m} \sum_{e \in \Gamma_{F}^{i}}\left\langle\nabla w \cdot s, c_{i}\right\rangle_{e}=\sum_{i=1}^{m} \sum_{e \in \Gamma_{F}^{i}} c_{i}\left(w\left(\nu_{e}^{1}\right)-w\left(\nu_{e}^{2}\right)\right)
\end{aligned}
$$

where $\nu_{e}^{j}, j=1,2$, represent respectively the first and last node of each edge $e$ along the boundary coordinate $s$.

Observing that $w$ is continuous and, by definition, null at the endpoints of each $\Gamma_{F}^{i}$, from (3.57) it follows

$$
(\varepsilon(\nabla w), \operatorname{Curl} \phi)_{\Omega}=0 .
$$

We therefore have

$$
\begin{aligned}
T_{3}= & \sum_{K \in \mathcal{C}_{h}}\left(\varepsilon\left(\nabla w_{h}\right), \operatorname{Curl} \phi\right)_{K} \\
= & \sum_{K \in \mathcal{C}_{h}}\left(\varepsilon\left(\nabla w_{h}\right), \operatorname{Curl}\left(\phi-\phi_{I I}\right)\right)_{K} \\
& +\sum_{K \in \mathcal{C}_{h}}\left(\varepsilon\left(\nabla w_{h}\right), \operatorname{Curl} \phi_{I I}\right)_{K},
\end{aligned}
$$


where $\phi_{I I}$ is the approximation of $\phi$ introduced in Definition 2. Integrating by parts triangle by triangle and recalling (3.24), we have

$$
\begin{aligned}
& \sum_{K \in \mathcal{C}_{h}}\left(\varepsilon\left(\nabla w_{h}\right), \operatorname{Curl}\left(\boldsymbol{\phi}-\boldsymbol{\phi}_{I I}\right)\right)_{K} \\
& =\sum_{K \in \mathcal{C}_{h}} \sum_{e \in \partial K}\left\langle\varepsilon\left(\nabla w_{h}\right) \boldsymbol{s}_{K}, \boldsymbol{\phi}-\phi_{I I}\right\rangle_{e}=0,
\end{aligned}
$$

where $\boldsymbol{s}_{K}$ represents the unit vector which is the counter clockwise rotation of $\boldsymbol{n}_{K}$ at each edge of $K \in \mathcal{C}_{h}$.

First integrating by parts and noting that $\operatorname{Curl} \boldsymbol{\phi}_{I I} \boldsymbol{n}_{e}$ is continuous across edges, then with a simple splitting, it follows

$$
\begin{aligned}
& \sum_{K \in \mathcal{C}_{h}}\left(\varepsilon\left(\nabla w_{h}\right), \operatorname{Curl} \phi_{I I}\right)_{K}=-\sum_{K \in \mathcal{C}_{h}} \sum_{e \in \partial K}\left\langle\nabla w_{h}, \operatorname{Curl} \boldsymbol{\phi}_{I I} \boldsymbol{n}_{K}\right\rangle_{e} \\
& =-\sum_{e \in \mathcal{E}_{h}}\left\langle\llbracket \nabla w_{h} \rrbracket, \operatorname{Curl} \boldsymbol{\phi}_{I I} \boldsymbol{n}_{e}\right\rangle_{e}=-\sum_{e \in \mathcal{E}_{h}^{i} \cup \mathcal{E}_{h}^{c}}\left\langle\llbracket \nabla w_{h} \rrbracket, \operatorname{Curl} \boldsymbol{\phi}_{I I} \boldsymbol{n}_{e}\right\rangle_{e} \\
& -\sum_{e \in \mathcal{E}_{h}^{s}}\left\langle\llbracket \nabla w_{h} \cdot \boldsymbol{s} \rrbracket,\left[\operatorname{Curl} \boldsymbol{\phi}_{I I}\right]_{\boldsymbol{n} \boldsymbol{s}}\right\rangle_{e}-\sum_{e \in \mathcal{E}_{h}^{f}}\left\langle\llbracket \nabla w_{h} \cdot \boldsymbol{s} \rrbracket,\left[\operatorname{Curl} \boldsymbol{\phi}_{I I}\right]_{\boldsymbol{n} \boldsymbol{s}}\right\rangle_{e} \\
& -\sum_{e \in \mathcal{E}_{h}^{s} \cup \mathcal{E}_{h}^{f}}\left\langle\llbracket \nabla w_{h} \cdot \boldsymbol{n} \rrbracket,\left[\operatorname{Curl} \boldsymbol{\phi}_{I I}\right]_{\boldsymbol{n n}}\right\rangle_{e}=T_{4}+T_{5}+T_{6}+T_{7} .
\end{aligned}
$$

Step 4. For the term $T_{4}$ above, first Hölder inequalities, then the Agmon and the inverse inequality, and finally the property (3.23) with $m=1$ give

$$
\begin{aligned}
& T_{4}=\sum_{e \in \mathcal{E}_{h}^{i} \cup \mathcal{E}_{h}^{c}}\left\langle\left(\llbracket \nabla w_{h} \rrbracket, \operatorname{Curl} \boldsymbol{\phi}_{I I} \boldsymbol{n}_{e}\right\rangle_{e}\right. \\
& \leq\left(\sum_{e \in \mathcal{E}_{h}^{i} \cup \mathcal{E}_{h}^{c}} h_{e}^{-1}\left\|\llbracket \nabla w_{h} \rrbracket\right\|_{L^{2}(e)}^{2}\right)^{1 / 2}\left(\sum_{e \in \mathcal{E}_{h}^{i} \cup \mathcal{E}_{h}^{c}} h_{e}\left\|\operatorname{Curl} \boldsymbol{\phi}_{I I} \boldsymbol{n}_{e}\right\|_{L^{2}(e)}^{2}\right)^{1 / 2} \\
& \leq C\left(\sum_{e \in \mathcal{E}_{h}^{i} \cup \mathcal{E}_{h}^{c}} h_{e}^{-1}\left\|\llbracket \nabla w_{h} \rrbracket\right\|_{L^{2}(e)}^{2}\right)^{1 / 2}\left(\sum_{K \in \mathcal{K}_{h}}\left\|\phi_{I I}\right\|_{H^{1}(K)}^{2}\right)^{1 / 2} \\
& \leq C\left(\sum_{e \in \mathcal{E}_{h}^{i} \cup \mathcal{E}_{h}^{c}} h_{e}^{-1}\left\|\llbracket \nabla w_{h} \rrbracket\right\|_{L^{2}(e)}^{2}\right)^{1 / 2}\|\boldsymbol{\phi}\|_{H^{1}(\Omega)} .
\end{aligned}
$$

Again a tangent and normal component splitting applied to (3.62) grants

$$
\begin{aligned}
T_{4} \leq & C\left(\sum_{e \in \mathcal{E}_{h}^{i} \cup \mathcal{E}_{h}^{c}} h_{e}^{-1}\left\|\llbracket \nabla w_{h} \rrbracket\right\|_{L^{2}(e)}^{2}\right)^{1 / 2}\|\boldsymbol{\phi}\|_{H^{1}(\Omega)} \\
\leq C & \left(\sum_{e \in \mathcal{E}_{h}^{i} \cup \mathcal{E}_{h}^{c}} h_{e}^{-1}\left\|\llbracket \nabla w_{h} \cdot \boldsymbol{n}_{e} \rrbracket\right\|_{L^{2}(e)}^{2}\right. \\
& \left.+\sum_{e \in \mathcal{E}_{h}^{i} \cup \mathcal{E}_{h}^{c}} h_{e}^{-1}\left\|\llbracket \nabla w_{h} \cdot \boldsymbol{s}_{e} \rrbracket\right\|_{L^{2}(e)}^{2}\right)^{1 / 2}\|\boldsymbol{\phi}\|_{H^{1}(\Omega)} .
\end{aligned}
$$


Observing that

$$
\llbracket \nabla w_{h} \cdot \boldsymbol{s}_{e} \rrbracket=\frac{\partial}{\partial s} \llbracket w_{h} \rrbracket \quad \forall e \in \mathcal{E}_{h},
$$

where $s$ represents the coordinate along the edge $e$, standard scaling arguments give

$$
\sum_{e \in \mathcal{E}_{h}^{i} \cup \mathcal{E}_{h}^{c}} h_{e}^{-1}\left\|\llbracket \nabla w_{h} \rrbracket \cdot s_{e}\right\|_{L^{2}(e)}^{2} \leq C \sum_{e \in \mathcal{E}_{h}^{i} \cup \mathcal{E}_{h}^{c}} h_{e}^{-3}\left\|\llbracket w_{h} \rrbracket\right\|_{L^{2}(e)}^{2} .
$$

Combining (3.63) with (3.65) it finally follows

$$
\begin{aligned}
T_{4} \leq C & \left(\sum_{e \in \mathcal{E}_{h}^{i} \cup \mathcal{E}_{h}^{c}} h_{e}^{-1}\left\|\llbracket \nabla w_{h} \cdot \boldsymbol{n}_{e} \rrbracket\right\|_{L^{2}(e)}^{2}\right. \\
& \left.+\sum_{e \in \mathcal{E}_{h}^{i} \cup \mathcal{E}_{h}^{c}} h_{e}^{-3}\left\|\llbracket w_{h} \rrbracket\right\|_{L^{2}(e)}^{2}\right)^{1 / 2}\|\boldsymbol{\phi}\|_{H^{1}(\Omega)} .
\end{aligned}
$$

The second term $T_{5}$ in (3.61) is bounded with identical arguments giving

$$
T_{5} \leq C\left(\sum_{e \in \mathcal{E}_{h}^{s}} h_{e}^{-3}\left\|\llbracket w_{h} \rrbracket\right\|_{L^{2}(e)}^{2}\right)^{1 / 2}\|\phi\|_{H^{1}(\Omega)} .
$$

We now observe that, along each edge $e \in \mathcal{E}_{h}$, the normal and tangent vectors are constant. Therefore, recalling Corollary 1, on each edge of $\Gamma_{S} \cup \Gamma_{F}$ it holds

$$
0=[\operatorname{Curl} \boldsymbol{\phi}]_{\boldsymbol{n} \boldsymbol{n}}=-[\nabla \boldsymbol{\phi} \boldsymbol{s}] \cdot \boldsymbol{n}=-\frac{\partial(\boldsymbol{\phi} \cdot \boldsymbol{n})}{\partial s},
$$

where $s$ represents, as usual, a coordinate along the edge. Again, using Corollary 1 and the same argument, we also have that

$$
\frac{\partial(\boldsymbol{\phi} \cdot \boldsymbol{s})}{\partial s} \text { is constant }
$$

on each edge of $\Gamma_{F}$. As a consequence of (3.68) and (3.69), we have that $\phi$ is piecewise linear on $\Gamma_{F}$ and that $\boldsymbol{\phi} \cdot \boldsymbol{n}$ is piecewise linear on $\Gamma_{S}$. Therefore we can apply (3.25) which, in combination with Corollary 1, easily gives

$$
\begin{aligned}
& {\left[\operatorname{Curl} \phi_{I I}\right]_{n \boldsymbol{n}}=[\operatorname{Curl} \phi]_{n \boldsymbol{n}}=0 \text { on } \Gamma_{S} \cup \Gamma_{F}} \\
& {\left[\operatorname{Curl} \phi_{I I}\right]_{\boldsymbol{n} \boldsymbol{s}}=[\operatorname{Curl} \phi]_{\boldsymbol{n} \boldsymbol{s}}=c_{i} \quad \text { on } \Gamma_{F}^{i}, i=1,2, \ldots, m \text {. }}
\end{aligned}
$$

Due to (3.70) we immediately get

$$
T_{7}=0,
$$

while (3.71), following the same argument used in (3.56)-(3.58), gives

$$
T_{6}=0 .
$$


Combining (3.59) with (3.60), (3.61), (3.66), (3.67), (3.72) and (3.73) we finally obtain

$$
\begin{aligned}
T_{3} \leq C & \left(\sum_{e \in \mathcal{E}_{h}^{i} \cup \mathcal{E}_{h}^{c}} h_{e}^{-1}\left\|\llbracket \nabla w_{h} \cdot \boldsymbol{n}_{e} \rrbracket\right\|_{L^{2}(e)}^{2}\right. \\
& \left.+\sum_{e \in \mathcal{E}_{h}^{i} \cup \mathcal{E}_{h}^{c} \cup \mathcal{E}_{h}^{s}} h_{e}^{-3}\left\|\llbracket w_{h} \rrbracket\right\|_{L^{2}(e)}^{2}\right)^{1 / 2}\|\boldsymbol{\phi}\|_{H^{1}(\Omega)} .
\end{aligned}
$$

Step 5. Combining (3.45) with (3.53), (3.54), (3.74) and recalling (3.49), gives

$$
\begin{aligned}
& \sum_{K \in \mathcal{C}_{h}}\left|e_{h}\right|_{H^{2}(K)}^{2} \\
& \leq C\left(\sum_{K \in \mathcal{C}_{h}} h_{K}^{4}\left\|f-f_{h}\right\|_{L^{2}(K)}^{2}+\sum_{K \in \mathcal{C}_{h}} \eta_{K}^{2}\right)^{1 / 2}\left(\sum_{K \in \mathcal{C}_{h}}\left|e_{h}\right|_{H^{2}(K)}\right)^{1 / 2},
\end{aligned}
$$

which implies (3.44) and hence (3.42) as well.

\subsection{Efficiency}

We have the following upper bound for the error estimator. The proof is not shown here since it is essentially identical to the efficiency proof of [6].

Theorem 2. Let $w$ be the solution of the problem (2.5) and $w_{h}$ the solution of the problem (2.8). Then it holds

$$
\eta_{K} \leq\left\|\left|w-w_{h}\left\|\left.\right|_{h, K}+h_{K}^{2}\right\| f-f_{h} \|_{L^{2}(K)},\right.\right.
$$

where ||$|\cdot| \|_{h, K}$ represents the local restriction of the norm $\||\cdot|\|_{h}$ to the triangle $K$ :

$$
\begin{aligned}
\|\| v\|\|_{h, K}^{2}= & |v|_{H^{2}(K)}^{2}+\sum_{e \in \partial K^{\prime}} c_{e} h_{e}^{-3}\|\llbracket v \rrbracket\|_{L^{2}(e)}^{2} \\
& +\sum_{e \in \partial K^{\prime \prime}} c_{e} h_{e}^{-1}\left\|\llbracket \nabla v \cdot \boldsymbol{n}_{e} \rrbracket\right\|_{L^{2}(e)}^{2} .
\end{aligned}
$$

where $\partial K^{\prime}$ and $\partial K^{\prime \prime}$ follow the definitions (3.3) and (3.4).

\section{Numerical results}

In this section, we present numerical results from various benchmark tests with different kinds of domains, boundary conditions and loadings. Our goal is to illustrate the reliability and robustness of the local error estimator by means of convergence graphs and mesh plots obtained from adaptively and uniformly refined computations.

In order to compare the numerical and theoretical results, we have chosen benchmark problems with known exact solutions, or at least the regularity 
of the exact solutions is known in a qualitative sense. In [7], the same benchmarks have been used for testing an a posteriori estimator of a $C^{0}$-continuous family of Kirchhoff plate elements.

For simplicity, as usual, in all of the test cases the values $E=1$ and $\nu=0.3$ have been used for the material constants.

\subsection{Adaptive solution strategies}

We have implemented Method 1 with the estimator of (3.1) in the opensource finite element software Elmer [18] developed for simulations of multiphysical problems. However, we emphasize that the error estimator proposed can be implemented in any adaptive finite element solver utilizing local error indicators.

For adaptive mesh refinements, the software supports the following methodology usually refered as error balancing strategy. First, a coarse starting mesh is prescribed for the problem considered. After the finite element solution and the corresponding error indicators are computed for the mesh, a complete remeshing is accomplished by Delaunay triangulations. The strategy for the refining-coarsening process is based on the local error indicators and on the assumption that the local error for an element $K$ is of the form

$$
\eta_{K}=C_{K} h_{K}^{p_{K}}
$$

with some constants $C_{K}$ and $p_{K}$. A new mesh is then built with the aim of having the error uniformly distributed over the elements of the new mesh.

We remark that the optimality of the final mesh depends on the desired maximal mesh density ratio as well as the stopping criteria given, i.e., the number of refinement steps and the global or local error tolerances. We have used the value 1.5 as the maximal mesh density ratio defining the change in the mesh density between two subsequent adaptive steps: For example, a subdomain currently covered by six elements is covered by four to nine elements after the next remeshing step.

\subsection{Convex rectangular domains - effectivity index}

For the first three problems of convex rectangular domains, the exact solution of each problem can be found as a trigonometric-hyperbolic series which we have used as a reference solution. For these problems, we compare the behavior of the estimated and true error, finally reported as the effectivity index, i.e., the ratio between these two error measures.

\subsubsection{Rectangle with simply supported boundaries}

We first consider the simply supported rectangle $\Omega=(0,1) \times(-1,1)$ with the uniform loading $f=1$. The exact solution for the problem can be found by writing the load as a trigonometric series as specified in [7]. The critical 
corner regularity in this problem is $w \in H^{3}(\Omega)[21,8]$, which implies, in the light of Proposition 1, the convergence rate $\mathcal{O}\left(h^{\sigma}\right)$ with $\sigma=1$.

The convergence graphs for uniformly refined meshes are shown in Figure 1 (left). The solid line represents the true error (circles), while the dashed line indicates the error estimator (asterisks). For clarity, the convergence rate $\mathcal{O}(h)$ is indicated in the same figure as well (dashed line without markers). We remark that for quasiuniform meshes it holds that $h \sim N^{-1 / 2}$, where $N$ denotes the number of elements in the mesh. The numerical results are in agreement with the theoretical ones: the behavior of the estimated error is almost identical with the true error, up to a multiplicative constant.

We note that for adaptively refined meshes the convergence graphs are practically identical to the present ones for uniform refinements. Since the exact solution of the problem is regular, this is in agreement with the theory. Hence, the graphs for adaptive refinements are omitted here and for the other benchmarks with convex domains below as well.

\subsubsection{Rectangle with simply supported and free boundaries}

Second, we consider the rectangle $\Omega=(0,1) \times(-1,1)$ with the simply supported left and right boundaries $\{x=0,-1 \leq y \leq 1\},\{x=1,-1 \leq y \leq 1\}$ and free bottom and top boundaries $\{y=-1,0 \leq x \leq 1\},\{y=1,0 \leq x \leq$ 1 . As above, the loading is constant, $f=1$, and again, the exact solution for the problem can be found by a trigonometric series, see [7]. The regularity in the corners is again $w \in H^{3}(\Omega)[21,8]$, which implies the convergence rate $\mathcal{O}\left(h^{\sigma}\right)=1$.

The convergence graphs for uniformly refined meshes are plotted in Figure 1 (right), with the solid line representing the true error (triangles), and the dashed line indicating the error estimator (asterisks). In this case, the true error and estimator are practically identical.

\subsubsection{Square with clamped boundaries}

Third, we consider the clamped square $\Omega=(-1,1) \times(-1,1)$ with the uniform loading $f=1$. In this case as well, the exact solution can be written as a trigometric series [7]. Now, the regularity in the corners is $w \in H^{4.74}(\Omega)$ $[21,8,9]$, which implies, by Proposition 1 , the convergence rate $\mathcal{O}\left(h^{\sigma}\right), \sigma=$ $\min \{2.74,1\}=1$.

The convergence graphs for uniformly refined meshes are shown in Figure 2 (left), together with the convergence rate $\mathcal{O}(h)$. Comparing the solid line with squares (true error) and the dashed line with asterisks (error estimator) clearly shows the identical behavior of the error measures.

\subsubsection{Effectivity index for the different problem types}

In figure 2 (right), the effectivity index for the adaptive error estimator, i.e., the ratio between the estimated and true error, is reported for the uniform refinements of the three test problems above. 
The dashed line in the figure represents the value 1 , the effectivity index lies between 0.6 and 2.9. In all of the test cases, the effectivity index first slightly decreases (between 8 and 22 elements in the mesh) and then uniformly remains in the range $0.6 \ldots 1.0$ (between 22 and 23218 elements). More precisely, after the first two steps, for the problem with simply supported boundaries (circles) the effectivity index remains around 0.6, while for the other two problems (squares, triangles) it stays around 1.0.

Finally, we note that the results here are similar to the ones in [7]. Altogether, within these different types of problems, the effectivity index remains on a certain almost constant level uniformly in the mesh size. Hence, together with the the theoretical results above, this seems to indicate that the error estimator can be used as an reliable and efficient error measure.
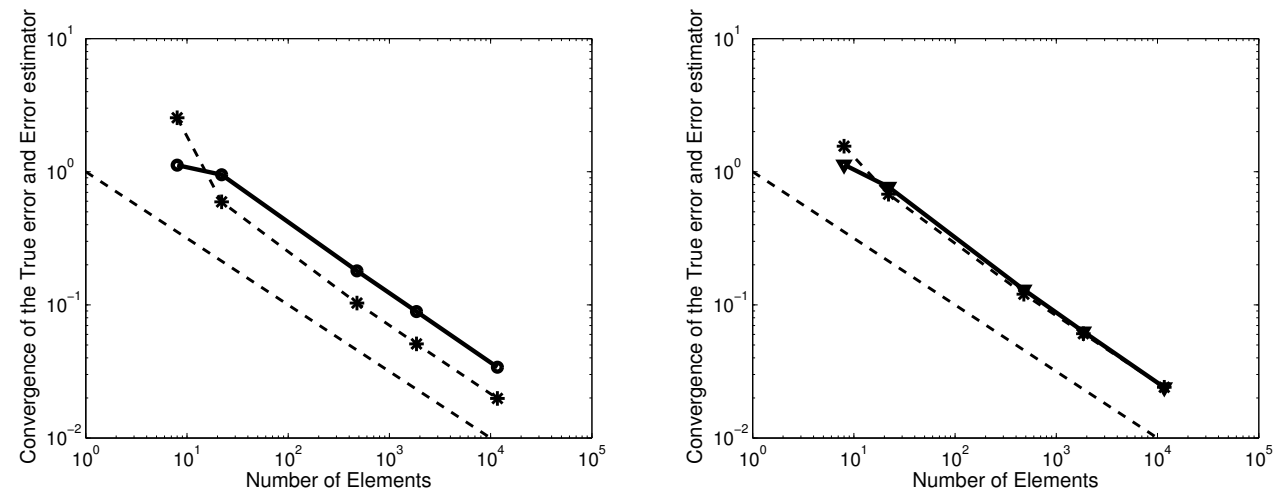

Figure 1: Left: Simply supported rectangle; convergence of the true error (solid line, circles) and the error estimator (dashed line, asterisks). Right: Simply supported and free rectangle; convergence of the true error (solid line, triangles) and the error estimator (dashed line, asterisks).
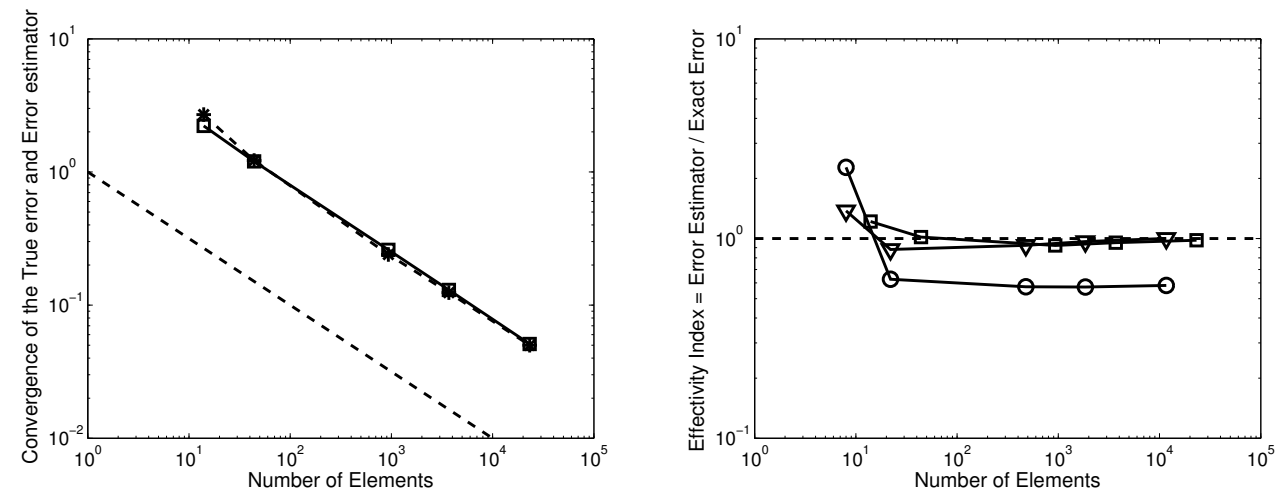

Figure 2: Left: Clamped square; convergence of the true error (solid line, squares) and the error estimator (dashed line, asterisks). Right: Effectivity index for uniform refinements; simply supported (circles), simply supported and free (triangles), clamped (squares) boundaries. 


\subsection{Non-convex domains}

Next, we consider a set of benchmark problems with nonconvex domains for which the behavior of the estimated error is reported alone, due to the lack of known exact solutions. However, the bahaviour of the exact solution is known in a qualitative sense. In particular, the regularity of the exact solution is known in the critical reentrant corners.

Within these problems which comprise different types of boundary conditions as well, we focus on comparing estimated errors of uniform and adaptive refinements.

\subsubsection{L-shaped domain with simply supported boundaries}

The first nonconvex benchmark problem is a uniformly loaded, $f=1$, simply supported plate of an L-shaped domain $\Omega$ with the corners $(0,0),(2,0),(2,1)$, $(1,1),(1,2)$ and $(0,2)$. The regularity in the critical L-corner is now $w \in$ $H^{7 / 3}(\Omega)[21,8]$, which implies the convergence rate $\mathcal{O}\left(h^{\sigma}\right), \sigma=\min \{1 / 3,1\}=$ $1 / 3$ for uniform refinements. We remark that without the corner singularity the convergence rate would be of order $\mathcal{O}(h)$.

In Figure 3, the convergence graphs are shown for both the uniformly (circles) and adaptively (triangles) refined meshes. The two upper graphs (solid lines) represent the global error estimator, while the lower ones (dashed lines) indicate the maximum local estimator. Moreover, the convergence rates $\mathcal{O}(h)$ and $\mathcal{O}\left(h^{1 / 3}\right)$ are presented in the same figure with dashed lines (without markers). Finally, two example meshes from adaptive steps with the distribution of the error estimator are depicted in Figures 7 and 8.

For analyzing the results, we first recall that for quasiuniform meshes it holds that $h \sim N^{-1 / 2}$, with $N$ denoting the number of elements in the mesh. Now, for the uniform refinements (circles), we see that the convergence rate first follows the order $\mathcal{O}(h)$ and then turns to the order $\mathcal{O}\left(h^{1 / 3}\right)$. This holds not only for the global error estimator but also for the maximum local one. The adaptive process (triangles), instead, starts as uniform refinements, but finally shows its robustness and finds the corner singularity and refines locally near the L-corner. This can be seen in the convergence graphs and in the meshes of Figures 7 and 8.

In addition to the corner singularity, the method refines the mesh near the simply supported boundaries as well. This is natural due to the boundary terms in the error estimator (3.2), which result from the fact that the essential boundary conditions of the problem are not fully a priori satisfied by the degrees of freedom of the discrete solution in the finite element space (2.7).

Compared to the results in [7], the refinements near the boundaries implied by the present method can be seen as the main difference. Otherwise, the results of these two methods are quite similar. 


\subsubsection{L-shaped domain with a clamped corner}

In this test problem, the two boundaries forming the reentrant corner of the L-shaped domain are clamped, while the remaining ones are again simply supported. The regularity in the critical corner is now $w \in H^{2.54}(\Omega)[21,8,9]$, which implies the convergence rate $\mathcal{O}\left(h^{\sigma}\right)$ with $\sigma=0.54$.

In Figure 4, the convergence graphs for the uniformly (circles) and adaptively (triangles) refined meshes are presented, together with the convergence rates $\mathcal{O}(h)$ and $\mathcal{O}\left(h^{0.54}\right)$ (dashed lines without markers). Two example meshes from adaptive steps with the error distribution are depicted in Figures 9 and 10.

Essentially the same comments as for the previous, completely simply supported L-shaped domain above apply for the results of this problem as well - except that now the corner singularity is weaker, which implies that, with respect to the refinement steps and the mesh density, the benefit from adaptivity comes true later than in the previous case.

\subsubsection{L-shaped domain with a free corner}

The two edges forming the reentrant corner of the L-shaped domain are now free, while the remaining ones are simply supported. The regularity in the corner is now $w \in H^{2.64}(\Omega)[21,8]$, which implies the convergence rate $\mathcal{O}\left(h^{\sigma}\right)$, $\sigma=\min \{0.64,1\}=0.64$.

For this case, the convergence graphs for the uniformly (circles) and adaptively (triangles) refined meshes are presented in Figure 4, with the convergence rates $\mathcal{O}(h)$ and $\mathcal{O}\left(h^{0.64}\right)$. Example meshes from adaptive steps are given in Figures 11 and 12.

Now, the corner singularity is once again weaker than before. Hence, in view of the convergence curves in Figure 5, the superiority of the adaptive computation is not so clear anymore. However, after some adaptive steps, the error and refinements still seem to concentrate near to the L-corner and the simply supported boundaries, see Figures 11 and 12.

\subsubsection{M-shaped domain with simply supported boundaries}

As the final test for the Morley element, we consider a uniformly loaded, $f=1$, M-shaped "corridor" domain with simply supported boundaries. The corner points of the domain $\Omega$ are now $(0,0),(1,0),(1,2.5),(2,1),(3,2.5)$, $(3,0),(4,0),(4,4),(3,4),(2,2.5),(1,4)$ and $(0,4)$.

The regularity in the critical $\mathrm{V}$-corner in the middle is $w \in H^{2.2}(\Omega)$, while in the two singular corners with widest opening $w \in H^{2.1}(\Omega)[21,8]$. This implies the convergence rate $\mathcal{O}\left(h^{\sigma}\right), \sigma=\min \{0.1,0.2,1\}=0.1$ for uniform refinements.

The convergence graphs for the uniformly (circles) and adaptively (triangles) refined meshes, together with the convergence rates $\mathcal{O}(h)$ and $\mathcal{O}\left(h^{0.1}\right)$, are now shown in Figure 6. The example meshes are shown in Figures 13 and 14 . 
In this case, there appears strong corner singularities of two different orders. The global convergence rate for the uniform refinements first follows the order $\mathcal{O}(h)$ of non-singularity, while for further refinements it turns to following the critical order $\mathcal{O}\left(h^{0.1}\right)$. In the first mesh example of Figure 13, the two strongest singularities are clearly visible. In the second mesh example of Figure 14, the method has found and evidently distinguished all of the three separate corner singularities.

Acknowledgements This work has been partly supported by Tekes - the Finnish Funding Agency for Technology and Innovation - through the project KOMASI, Modelling and simulation of coupled problems in mechanics and electrical engineering (decision number 40322/07). The computations of this work has been accomplished in the computing environment of CSC - the Finnish IT Center for Science. 


\section{Appendix}

Let $v$ indicate a sufficiently regular scalar field $\Omega \rightarrow \mathbb{R}$. Analogously, let $\phi$ and $\boldsymbol{\sigma}$ represent, respectively, a vector field $\Omega \rightarrow \mathbb{R}^{2}$ and a second order tensor field $\Omega \rightarrow \mathbb{R}^{2 \times 2}$, both sufficiently regular. Finally, a subindex $i$ after a comma will indicate a derivative with respect to the coordinate $x_{i}, i=1,2$.

We then have the following definitions for the differential operators:

$$
\begin{aligned}
& \nabla v=\left(\begin{array}{l}
v_{, 1} \\
v_{, 2}
\end{array}\right), \quad \operatorname{curl} v=\left(\begin{array}{c}
-v_{, 2} \\
v_{, 1}
\end{array}\right), \\
& \nabla \boldsymbol{\phi}=\left(\begin{array}{ll}
\phi_{1,1} & \phi_{1,2} \\
\phi_{2,1} & \phi_{2,2}
\end{array}\right), \quad \operatorname{Curl} \boldsymbol{\phi}=\left(\begin{array}{ll}
-\phi_{1,2} & \phi_{1,1} \\
-\phi_{2,2} & \phi_{2,1}
\end{array}\right), \\
& \operatorname{div} \boldsymbol{\phi}=\phi_{1,1}+\phi_{2,2}, \quad \operatorname{rot} \boldsymbol{\phi}=\phi_{2,1}-\phi_{1,2}, \\
& \operatorname{div} \boldsymbol{\sigma}=\left(\begin{array}{l}
\sigma_{11,1}+\sigma_{12,2} \\
\sigma_{21,1}+\sigma_{22,2}
\end{array}\right), \quad \operatorname{rot} \boldsymbol{\sigma}=\left(\begin{array}{c}
\sigma_{12,1}-\sigma_{11,2} \\
\sigma_{22,1}-\sigma_{21,2}
\end{array}\right) .
\end{aligned}
$$

Furthermore, the strain tensor is defined as the symmetric gradient,

$$
\varepsilon(\boldsymbol{\phi})=\left(\begin{array}{cc}
\phi_{1,1} & \frac{\phi_{1,2}+\phi_{2,1}}{2} \\
\frac{\phi_{1,2}+\phi_{2,1}}{2} & \phi_{2,2}
\end{array}\right) .
$$

The corresponding formula for integration by parts are, for a scalar $v$ and a vector $\phi$,

$$
\begin{aligned}
(\nabla v, \boldsymbol{\phi})_{\Omega} & =-(v, \operatorname{div} \boldsymbol{\phi})_{\Omega}+\langle v, \boldsymbol{\phi} \cdot \boldsymbol{n}\rangle_{\partial \Omega}, \\
(\operatorname{curl} v, \boldsymbol{\phi})_{\Omega} & =-(v, \operatorname{rot} \boldsymbol{\phi})_{\Omega}+\langle v, \boldsymbol{\phi} \cdot \boldsymbol{s}\rangle_{\partial \Omega},
\end{aligned}
$$

and for a vector $\phi$ and a tensor $\boldsymbol{\sigma}$,

$$
\begin{aligned}
(\boldsymbol{\nabla} \phi, \boldsymbol{\sigma})_{\Omega} & =-(\boldsymbol{\phi}, \operatorname{div} \boldsymbol{\sigma})_{\Omega}+\langle\boldsymbol{\phi}, \boldsymbol{\sigma n}\rangle_{\partial \Omega} \\
(\operatorname{Curl} \phi, \boldsymbol{\sigma})_{\Omega} & =-(\boldsymbol{\phi}, \operatorname{rot} \boldsymbol{\sigma})_{\Omega}+\langle\boldsymbol{\phi}, \boldsymbol{\sigma} \boldsymbol{s}\rangle_{\partial \Omega}
\end{aligned}
$$




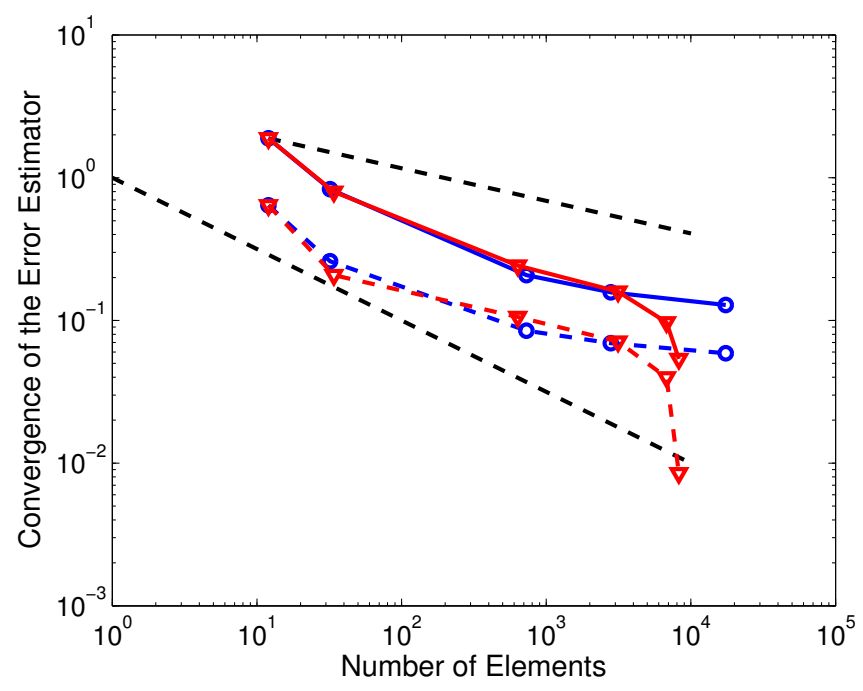

Figure 3: Simply supported L-corner: Convergence of the global estimator (solid lines) and the maximum local estimator (dashed lines); Circles for the uniform refinements, triangles for the adaptive refinements.

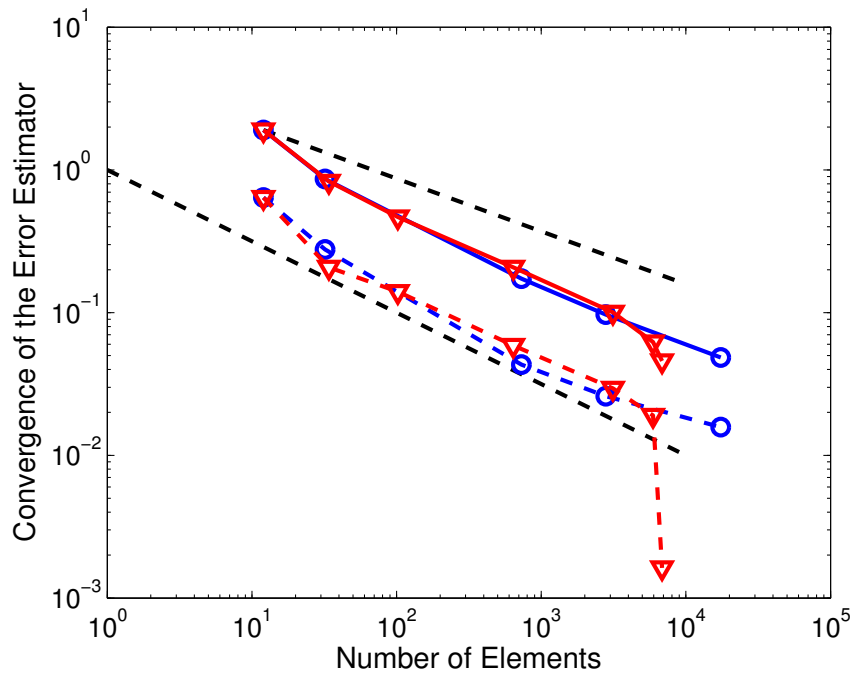

Figure 4: Clamped L-corner: Convergence of the global estimator (solid lines) and the maximum local estimator (dashed lines); Circles for the uniform refinements, triangles for the adaptive refinements. 


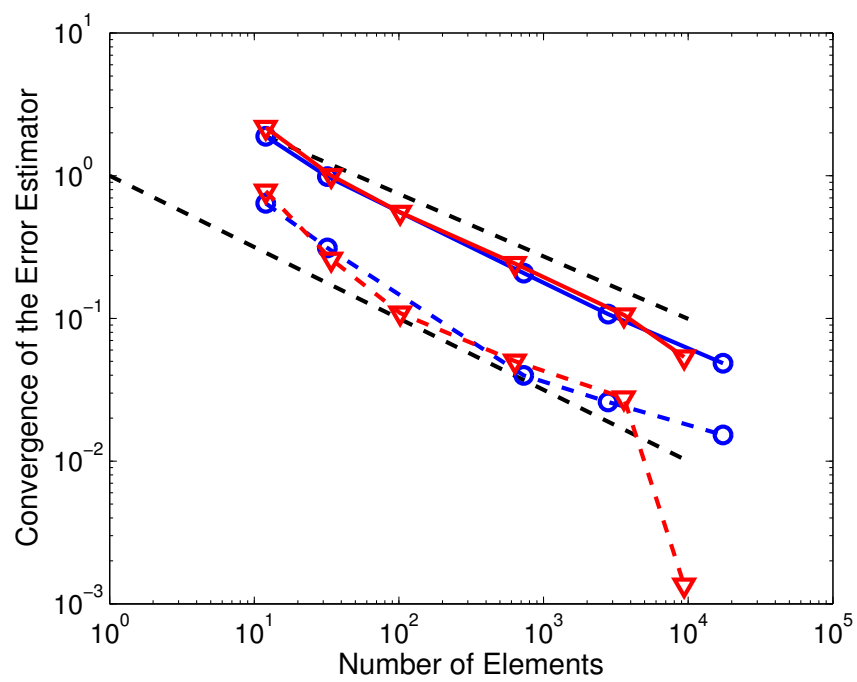

Figure 5: Free L-corner: Convergence of the global estimator (solid lines) and the maximum local estimator (dashed lines); Circles for the uniform refinements, triangles for the adaptive refinements.

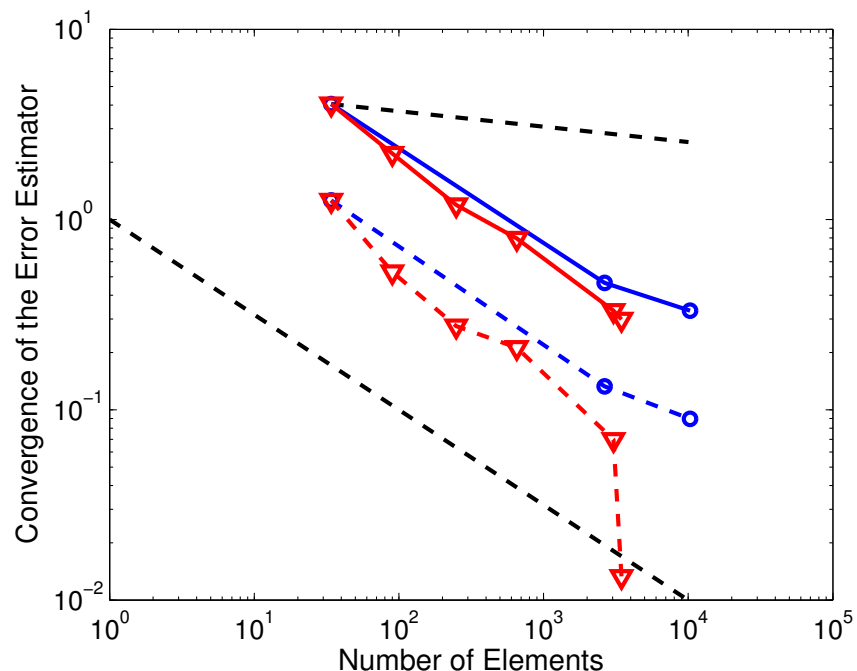

Figure 6: Simply supported M-domain: Convergence of the global estimator (solid lines) and the maximum local estimator (dashed lines); Circles for the uniform refinements, triangles for the adaptive refinements. 


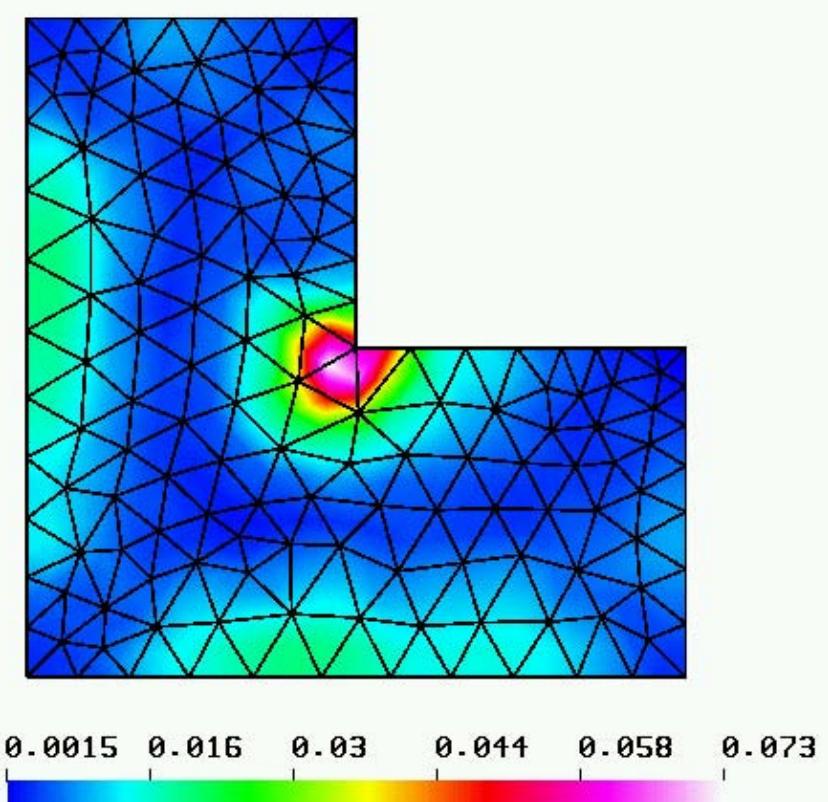

Figure 7: Simply supported L-corner: Distribution of the error estimator for the step 3 of the refinement process.
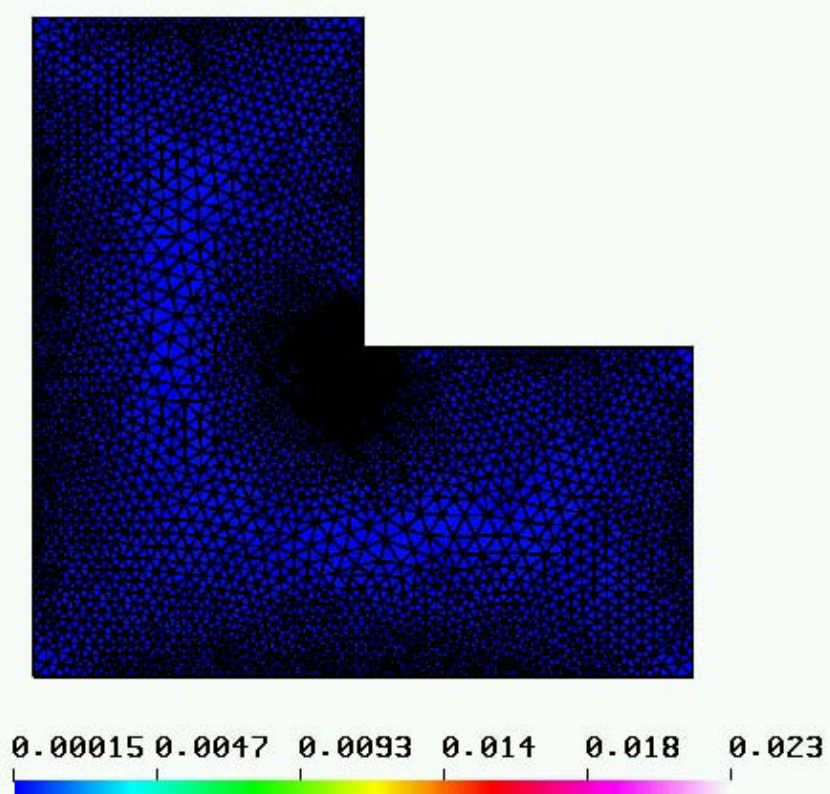

Figure 8: Simply supported L-corner: Distribution of the error estimator for the step 11 of the refinement process. 

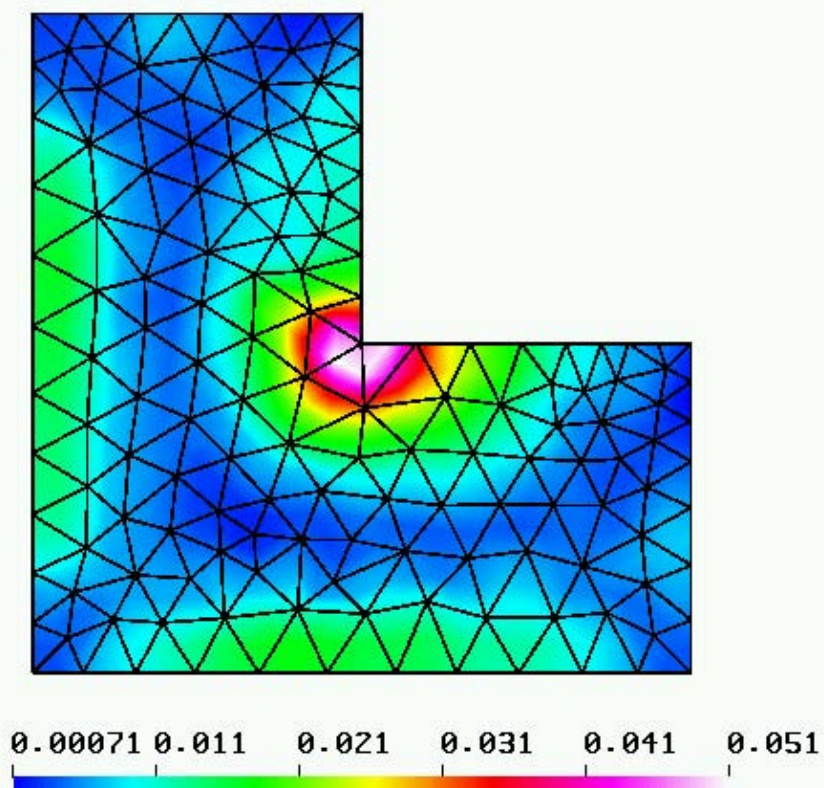

Figure 9: Clamped L-corner: Distribution of the error estimator for the step 3 of the refinement process.
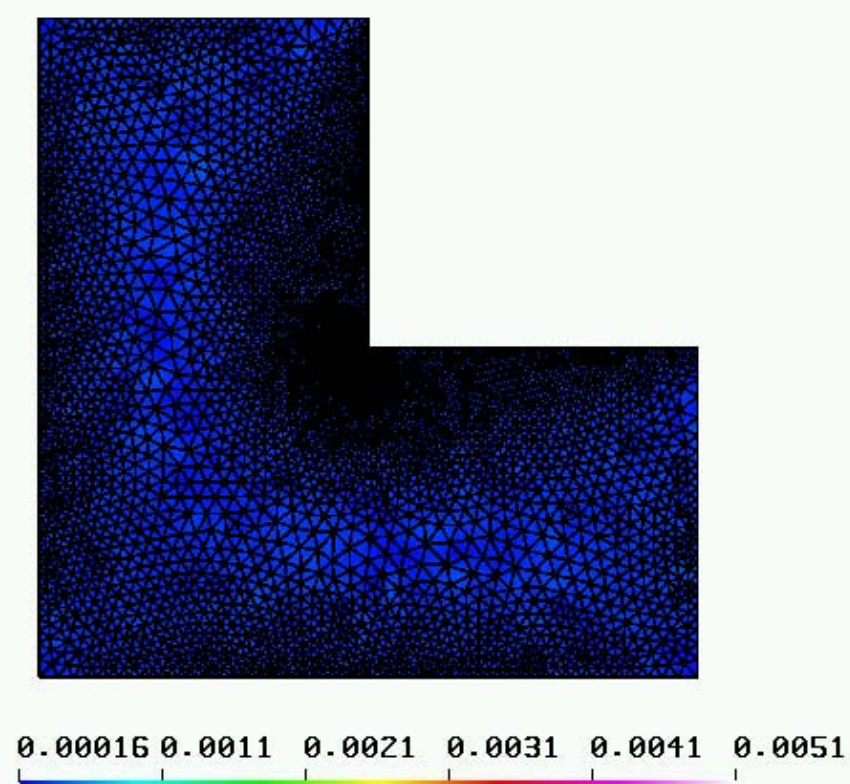

Figure 10: Clamped L-corner: Distribution of the error estimator for the step 11 of the refinement process. 

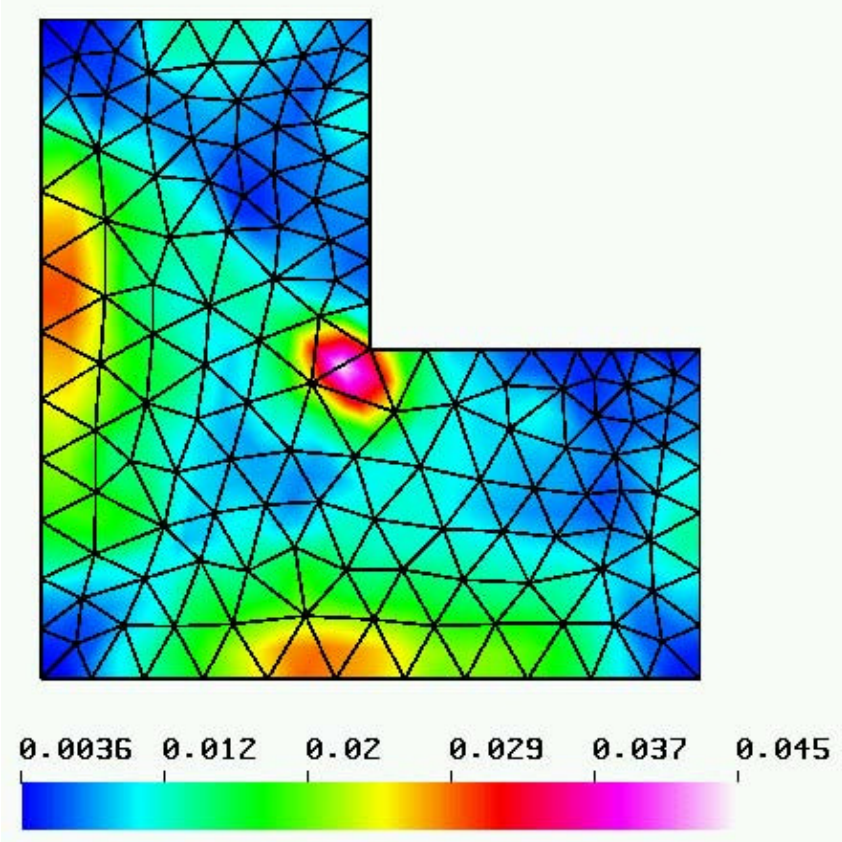

Figure 11: Free L-corner: Distribution of the error estimator for the step 3 of the refinement process.
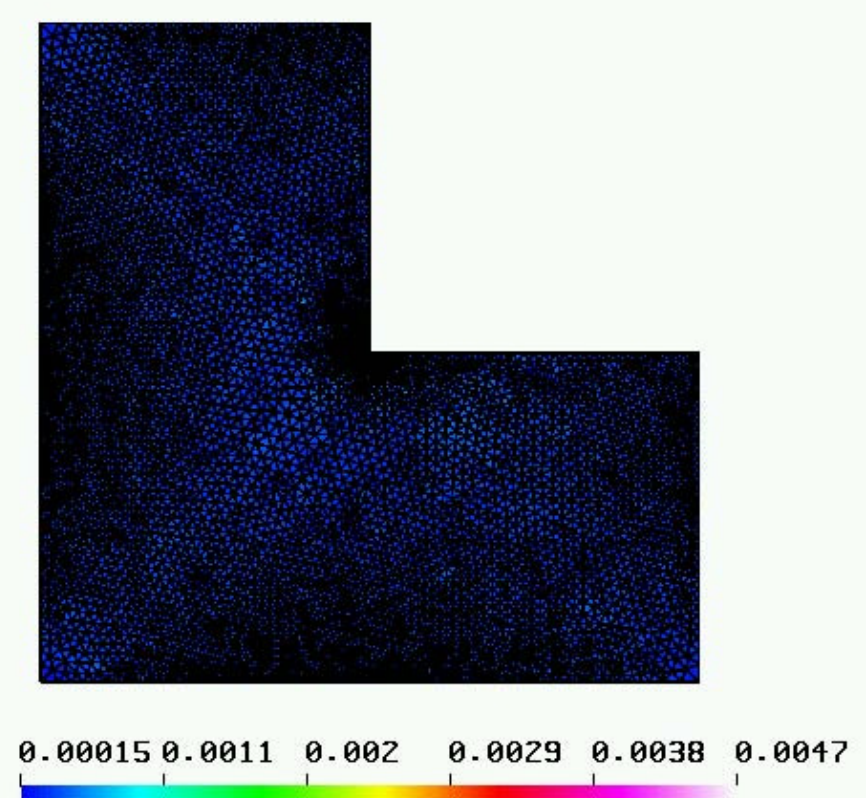

Figure 12: Free L-corner: Distribution of the error estimator for the step 11 of the refinement process. 


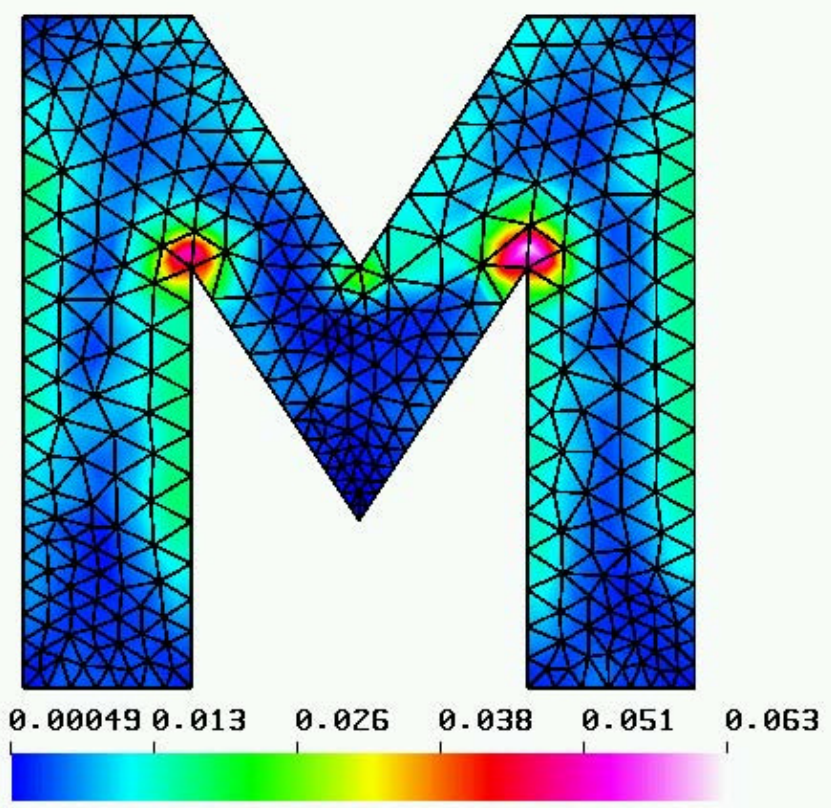

Figure 13: Simply supported M-domain: Distribution of the error estimator for the step 3 of the refinement process.

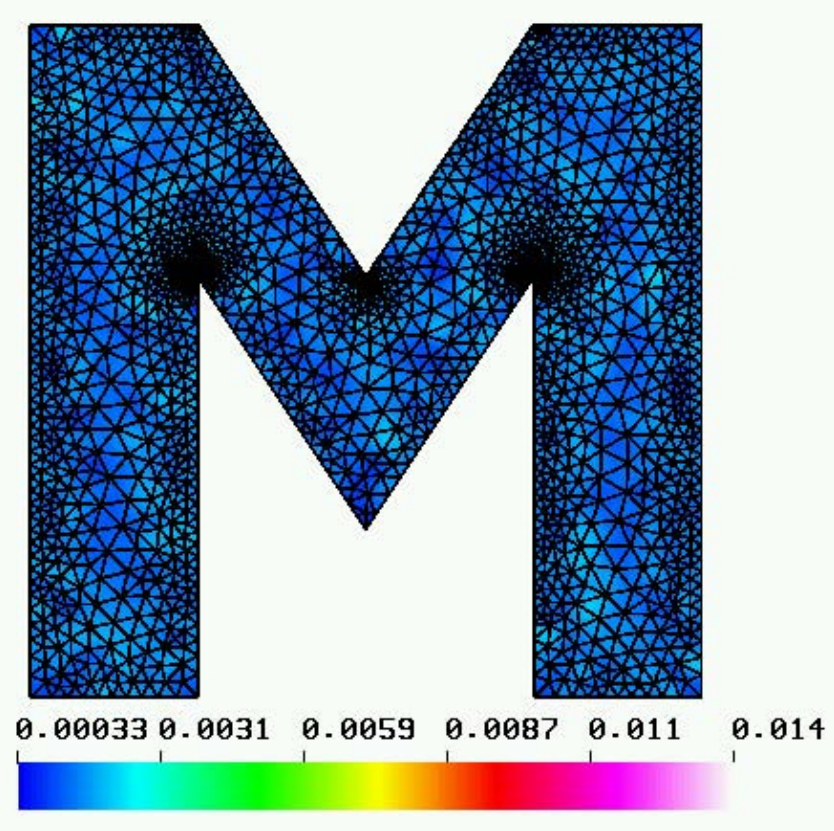

Figure 14: Simply supported M-domain: Distribution of the error estimator for the step 11 of the refinement process. 


\section{References}

[1] S. Agmon. Lectures on Elliptic Boundary Value Problems. Van Nostrand, Princeton, NJ, 1965.

[2] J.-L. Batoz, K.-J. Bathe, and L. W. Ho. A study of three-node triangular plate bending elements. Int. J. Num. Meths. Eng., 15:1771-1812, 1980.

[3] J. L. Batoz, C. L. Zheng, and F. Hammadi. Formulation and evaluation of new triangular, quadrilateral, pentagonal and hexagonal discrete Kirchhoff plate/shell elements. Int. J. Num. Meths. Eng., 52:615-630, 2001.

[4] L. Beirão da Veiga. Finite element methods for a modified ReissnerMindlin free plate model. SIAM J. Num. Anal., 42:1572-1591, 2004.

[5] L. Beirão da Veiga, J. Niiranen, and R. Stenberg. A family of $C^{0}$ finite elements for Kirchhoff plates I: Error analysis. SIAM J. Num. Anal., 45:2047-2071, 2007.

[6] L. Beirão da Veiga, J. Niiranen, and R. Stenberg. A posteriori error estimates for the Morley plate bending element. Numer. Math., 106:165179, 2007.

[7] L. Beirão da Veiga, J. Niiranen, and R. Stenberg. A family of $C^{0}$ finite elements for Kirchhoff plates II: Numerical results. Comp. Meths. Appl. Mech. Engrg., 197:1850-1864, 2008.

[8] H. Blum and R. Rannacher. On the boundary value problem of the biharmonic operator on domains with angular corners. Math. Meth. Apll. Sci., 2:556-581, 1980.

[9] J. H. Bramble, J. E. Pasciak, and C. Bacuta. Shift theorems for the biharmonic Dirichlet problem. In Recent Progress in Computational and Applied PDEs, pages 1-26, New York, 2002. Kluwer Academic/Plenum Publishers.

[10] S. C. Brenner and L. R. Scott. The Mathematical Theory of Finite Element Methods. Springer, New York, 2008.

[11] A. Charbonneau, K. Dossou, and R. Pierre. A residual-based a posteriori error estimator for the Ciarlet-Raviart formulation of the first biharmonic problem. Num. Meth. Part. Diff. Eq., 13:93-111, 1997.

[12] P. G. Ciarlet. The Finite Element Method for Elliptic Problems. NorthHolland, Amsterdam, 1987.

[13] P. G. Ciarlet. Mathematical Elasticity - Volume II: Theory of Plates. Elsevier, Amsterdam, 1997. 
[14] P. Destuynder and T. Nevers. A new finite element scheme for bending plates. Comp. Meths. Appl. Mech. Engrg., 68:127-139, 1988.

[15] P. Destuynder and T. Nevers. Une modification du modèle de Mindlin pour les plaques minces en flexion présentant un bord libre. RAIRO Modél. Math. Anal. Numér., 22:217-242, 1988.

[16] P. Destuynder and M. Salaun. Mathematical Analysis of Thin Plate Models. Springer-Verlag, Berlin, 1996.

[17] G. Engel, K. Garikipati, T. J. R. Hughes, M. G. Larson, L. Mazzei, and R. L. Taylor. Continuous/discontinuous finite element approximations of fourth-order elliptic problems in structural and continuum mechanics with applications to thin beams and plates, and strain gradient elasticity. Comp. Meths. Appl. Mech. Engrg., 191:3669-3750, 2002.

[18] Elmer finite element software homepage. http://www.csc.fi/elmer.

[19] T. Grätsch and K.-J. Bathe. A posteriori error estimation techniques in practical finite element analysis. Comp. Struct., 83:235-265, 2005.

[20] M. Lyly, J. Niiranen, and R. Stenberg. Superconvergence and postprocessing of MITC plate elements. Comp. Meths. Appl. Mech. Engrg., 196:3110-3126, 2007.

[21] H. Melzer and R. Rannacher. Spannungskonzentrationen in Eckpunkten der vertikal belasteten Kirchhoffschen Platte. Bauingenieur, 55:181-189, 1980 .

[22] W. Ming and J. Xu. The Morley element for fourth order elliptic equations in any dimensions. Numer. Math., 103:155-169, 2006.

[23] L. S. D. Morley. The triangular equilibrium element in the solution of plate bending problems. Aero. Quart., 19:149-169, 1968.

[24] P. Neittaanmäki and S. Repin. A posteriori error estimates for boundary value problems related to the biharmonic operator. East-West J. Numer. Math., 9:157-178, 2001.

[25] J. Nečas and I. Hlaváček. Mathematical Theory of Elastic and ElastoPlastic Bodies: An Introduction. Elsevier, Amsterdam, 1981.

[26] L. R. Scott and S. Zhang. Finite element interpolation of nonsmooth functions satisfying boundary conditions. Math. Comp., 54:483-493, 1990.

[27] Z. C. Shi. Error estimates for the Morley element. Chinese J. Numer. Math. Appl., 12:102-108, 1990. 

(continued from the back cover)

A551 István Faragó, Janos Karatson, Sergey Korotov

Discrete maximum principles for the FEM solution of some nonlinear parabolic problems

August 2008

A550 István Faragó, Róbert Horváth, Sergey Korotov

Discrete maximum principles for FE solutions of nonstationary

diffusion-reaction problems with mixed boundary conditions

August 2008

A549 Antti Hannukainen, Sergey Korotov, Tomás Vejchodský

On weakening conditions for discrete maximum principles for linear finite element schemes

August 2008

A548 Kalle Mikkola

Weakly coprime factorization, continuous-time systems, and strong- $H^{p}$ and Nevanlinna fractions

August 2008

A547 Wolfgang Desch, Stig-Olof Londen

A generalization of an inequality by N. V. Krylov

June 2008

A546 Olavi Nevanlinna

Resolvent and polynomial numerical hull

May 2008

A545 Ruth Kaila

The integrated volatility implied by option prices, a Bayesian approach

April 2008

A544 Stig-Olof Londen, Hana Petzeltová

Convergence of solutions of a non-local phase-field system

March 2008

A543 Outi Elina Maasalo

Self-improving phenomena in the calculus of variations on metric spaces

February 2008 
HELSINKI UNIVERSITY OF TECHNOLOGY INSTITUTE OF MATHEMATICS RESEARCH REPORTS

The reports are available at http://math.tkk.fi/reports/ .

The list of reports is continued inside the back cover.

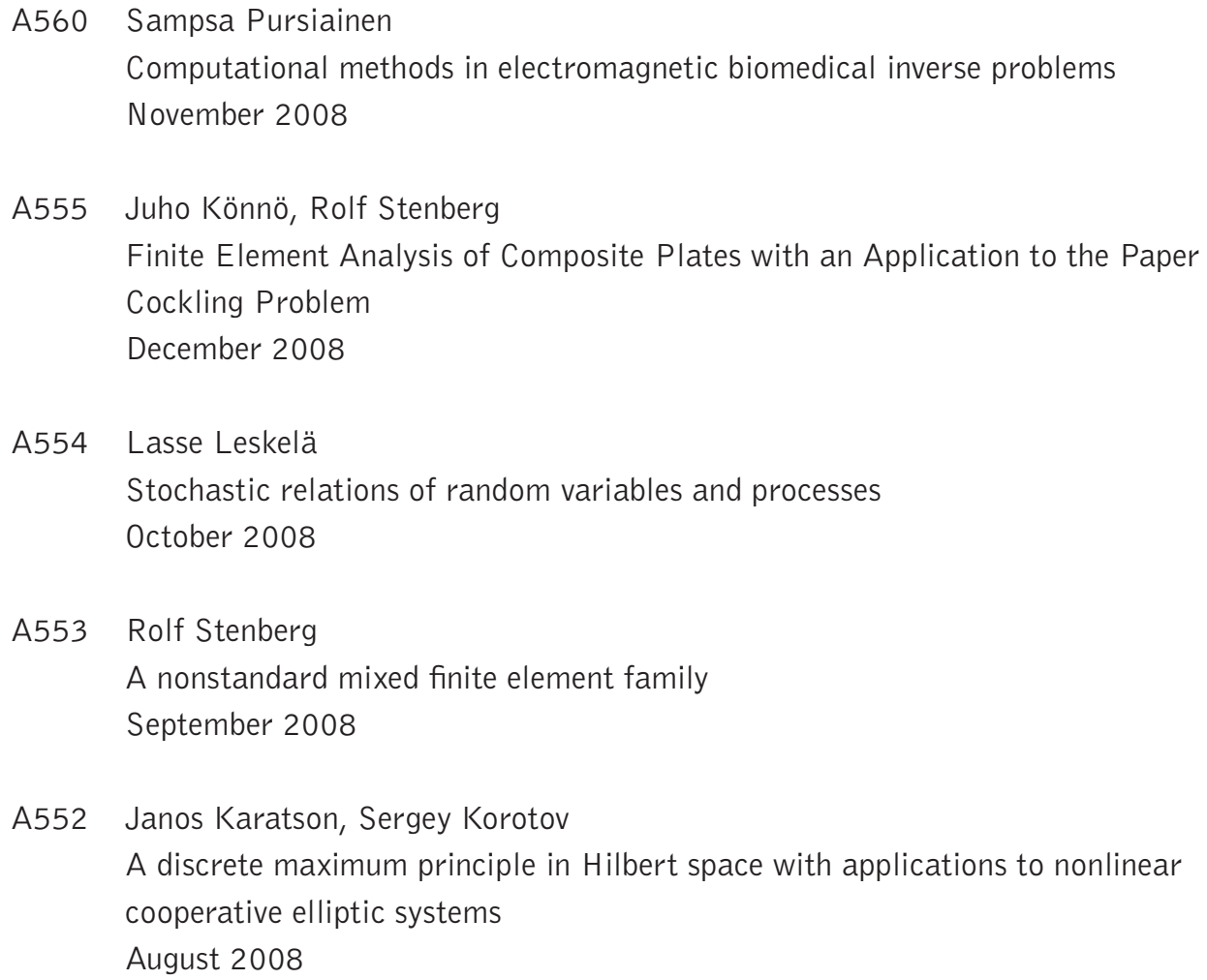

ISBN 978-951-22-9602-6 (print)

ISBN 978-951-22-9603-3 (PDF) 